\title{
18. DISTRIBUTION, CHEMICAL COMPOSITION, AND SOURCES OF OLIGOCENE TO MIOCENE VOLCANIC ASHES FROM SITES 907, 908, AND 913 ${ }^{1}$
}

\author{
Reinhard Werner, ${ }^{2}$ Hans-Joachim Wallrabe-Adams, ${ }^{2}$ Christian Lacasse, ${ }^{3}$ Hans-Ulrich Schmincke, ${ }^{2}$ and Jörn Thiede ${ }^{2}$
}

\begin{abstract}
The volcanogenic component of 54 ash layers and ash-bearing sediments recovered at Sites 907, 908, and 913 (Ocean Drilling Program Leg 151) in the Greenland-Iceland-Norwegian Seas (Iceland Plateau, Greenland Basin, Hovgård Ridge) consists mainly of colorless and/or light to dark brown glass particles with an average medium grain size of $100 \mu \mathrm{m}$ ( $\pm 60 \mu \mathrm{m})$; crystals and lithics are minor constituents. Colorless glass shards are primarily bubble wall shards and pumice fragments. Most light to dark-brown glass shards are slightly vesicular or dense blocky shards, but highly vesicular or pumice-like fragments also occur. The compositions of glass shards comprise subalkalic and low-K subalkalic basaltic ( $27 \%$ of all analyzed shards), basalticandesitic (9\%), andesitic $(6 \%)$, dacitic $(<1 \%)$, and trachytic $(4 \%)$, as well as low-K and high- $\mathrm{K}$ rhyolitic $(47 \%$ and $7 \%$, respectively).

The rhyolitic and trachytic tephra deposits can be attributed to highly explosive Plinian eruptions. Some mafic or intermediate ash deposits of Hole 907A show the influence of hydroclastic fragmentation processes, suggesting submarine or subglacial/ sublacustrine eruption environments. Major element compositions suggest that most ash layers are derived from Iceland and to a lesser extent from the Jan Mayen System. The oldest basaltic ashes of Hole 907A possibly originate from submarine eruptions at the ancient Kolbeinsey Ridge as indicated by their particle shape and the low degree of degassing.

The well-preserved record of middle to late Miocene ash deposits in Hole $907 \mathrm{~A}$ indicates a short but strong increase in explosive volcanic activity during the late middle Miocene but relatively low activity rates for early middle Miocene and late Miocene.
\end{abstract}

\section{INTRODUCTION}

Numerous discrete ash layers and ash-bearing sediments recovered during Leg 151 (North Atlantic Arctic Gateways) at Sites 907, 908, and 913 reflect Cenozoic volcanic activity in the North Atlantic region north of Iceland. Site 907 is located on the Iceland Plateau between the active spreading center of the Kolbeinsey Ridge and the continental Jan Mayen Ridge. Site 908 was drilled on the Hovgård Ridge southwest of Svalbard, and Site 913 is situated in the Greenland Basin (Fig. 1). Here, we report volcanological and geochemical studies of ash deposits in Holes 908A and 913B as well as the Miocene sequence of Hole 907A. The Pliocene and Quaternary tephra in Hole 907A are discussed by Lacasse et al. (this volume).

The sedimentary sequence of Hole $907 \mathrm{~A}\left(69^{\circ} 14.9^{\prime} \mathrm{N}, 12^{\circ} 41.8^{\prime} \mathrm{W}\right.$, water depth $1800 \mathrm{~m}$, penetration $224.1 \mathrm{~m}$, recovery $102 \%$ ) spans the last $14 \mathrm{Ma}$ from middle Miocene to Holocene and contains the majority of the ash deposits drilled on Leg 151 (Fig. 2). Almost 30 distinct Miocene ash layers ( $>1 \mathrm{~cm}$ thick) were recorded by the Shipboard Scientific Party (1995a) in lithologic Subunit IIIB and Unit IV (Cores 151-907A-11H to $21 \mathrm{H}$ ). Additional layers were identified by downhole measurements of GRAPE-density and whole core magnetic susceptibility (Rack et al., this volume). Altogether 51 Miocene ash deposits from Hole 907A were studied.

Hole $913 \mathrm{~B}\left(75^{\circ} 29.35^{\prime} \mathrm{N}, 6^{\circ} 56.81^{\prime} \mathrm{E}\right.$, water depth $3330 \mathrm{~m}$, penetration $770.3 \mathrm{~m}$, core recovery $32 \%$ ) ranges in age from early Eocene to

Thiede, J., Myhre, A.M., Firth, J.V., Johnson, G.L., and Ruddiman, W.F. (Eds.), 1996. Proc. ODP, Sci. Results, 151: College Station, TX (Ocean Drilling Program).

${ }^{2}$ GEOMAR, Research Center for Marine Geosciences, Wischhofstraße 1-3, D24148 Kiel, Federal Republic of Germany. rwerner@ geomar.de

${ }^{3}$ Graduate School of Oceanography, University of Rhode Island, Narragansett Bay Campus, Narragansett, RI 02882-1197, U.S.A.
Holocene. Two ash layers in lithologic Subunit IIIB at 463.9 and 486.3 meters below sea floor (mbsf) are late Eocene to early Oligocene in age, the oldest tephra drilled on Leg 151.

Hole $908 \mathrm{~A}\left(78^{\circ} 23.112^{\prime} \mathrm{N}, 1^{\circ} 21.637^{\prime} \mathrm{E}\right.$, water depth of $1273.5 \mathrm{~m}$, penetration $344.6 \mathrm{~m}$, core recovery $91.1 \%$ ) contains only one ash layer in lithologic Subunit IC at 154.7 mbsf (late Miocene to early Pliocene).

The aim of this study was to characterize Cenozoic volcanic processes in the northern North Atlantic area within the scope of plate tectonics. Major element compositions indicate potential source regions for the tephra. We also evaluated transport and depositional processes as well as the paleoenvironment at the time of eruption using textural criteria and volatile contents of glass particles. Some conclusions regarding the temporal evolution of volcanic activity during Miocene in the northern North Atlantic are also made.

\section{METHODS}

According to shipboard descriptions (VCDs, barrel sheets, smear slide descriptions) 52 ash layers and ash-bearing sediments were sampled at one or two depth intervals (top and bottom) at the Bremen Core Repository. Thirty-eight additional horizons possibly containing some ash could not be identified visually and were sampled on the basis of whole core magnetic susceptibility measurements and GRAPE bulk density data of the sediments (Rack et al., this volume). In our experience, most peaks in the magmatic susceptibility plots with values exceeding $80-100 \times 10^{-6} \mathrm{cgs}$ indicate ash-rich horizons. Thin sections were made from all samples received. Fifty-four samples containing fresh glass particles were selected for detailed studies.

Grain size and sorting of glass shards, morphology, color, and lithic and crystal components and their abundances were determined 


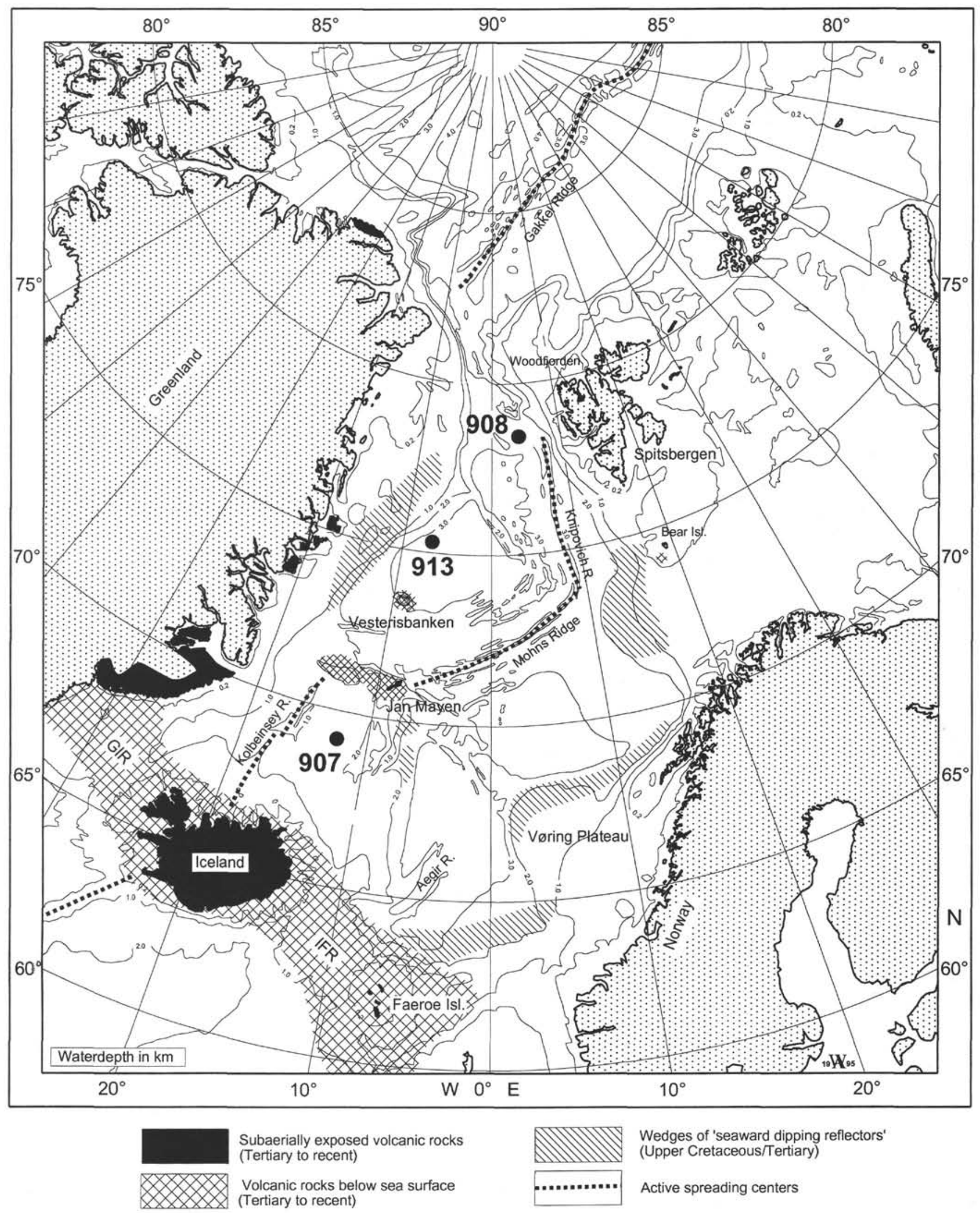

Figure 1. Locations of Sites 907, 908, and 913, and the generalized distribution of the Tertiary to Holocene volcanic activities in the North Atlantic Volcanic Province. Compiled from different sources (e.g., Mutter et al., 1988; Eldholm, 1991; Mørk and Duncan, 1993). GIR = Greenland-Iceland Ridge, IFR = IcelandFaeroe Ridge (= Greenland-Scotland Ridge). 

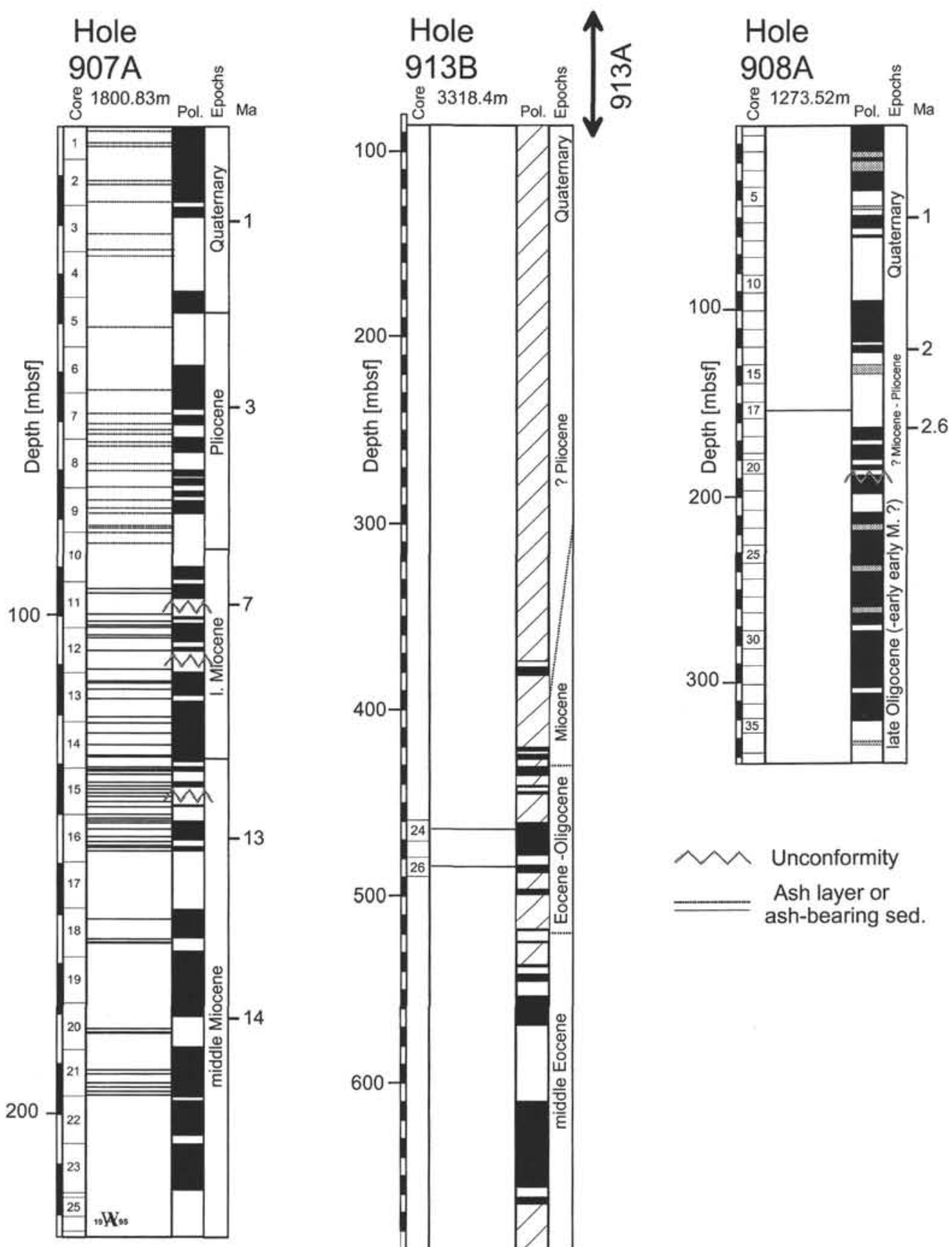

Figure 2. Stratigraphic distribution of ash layers and ash-bearing sediments in Holes 907A, 908A, and 913B. Ash deposits indicated by dashed lines (Site 907A, Pliocene-Quaternary) are described by Lacasse et al. (this volume). Magnetozones from Shipboard Scientific Party (1995a-c): black = normal polarity; white = reversed polarity; gray $=$ indeterminate polarity.

in thin sections. Maximum and medium diameter of 50-80 individual glass particles, in addition to the five largest shards, were measured in each thin section using calibrated ocular scales. The grain size data presented in this paper are the arithmetic means of these measurements. Abundance was estimated by counting about 300 grains in each thin section (Table 1).

Major element compositions and volatile contents $(\mathrm{S}, \mathrm{Cl}$, and $\mathrm{F})$ of fresh glass particles were determined using a CAMECA SX50 electron microprobe. The operation conditions were an accelerating voltage of $15 \mathrm{kV}$ and $10 \mathrm{nA}$ beam current. To minimize Na loss, silicic glasses were analyzed with the beam rastered (TV mode) over an area of $13 \times 9 \mu \mathrm{m}$ (magnification $10,000 \times$ ). Major element contents obtained for the homogeneous LIPARI standard (obsidian, Lipari, Cannetto Lami lava) are consistent with data reported by Hunt and Hill (1993) and with an X-ray fluorescence (XRF) analysis (Table 2). Besides the LIPARI standard, the natural glass standards JDF-D2 (MORB-glass) were used for calibration. Several additional basaltic and rhyolitic glass standards of known composition (e.g., KN 18, 
Table 1. Type, stratigraphic setting, textural characteristics, and composition of ash layers and ash-bearing sediments recovered at Holes $907 \mathrm{~A}$ (Miocene sequence), 908A, and 913B.

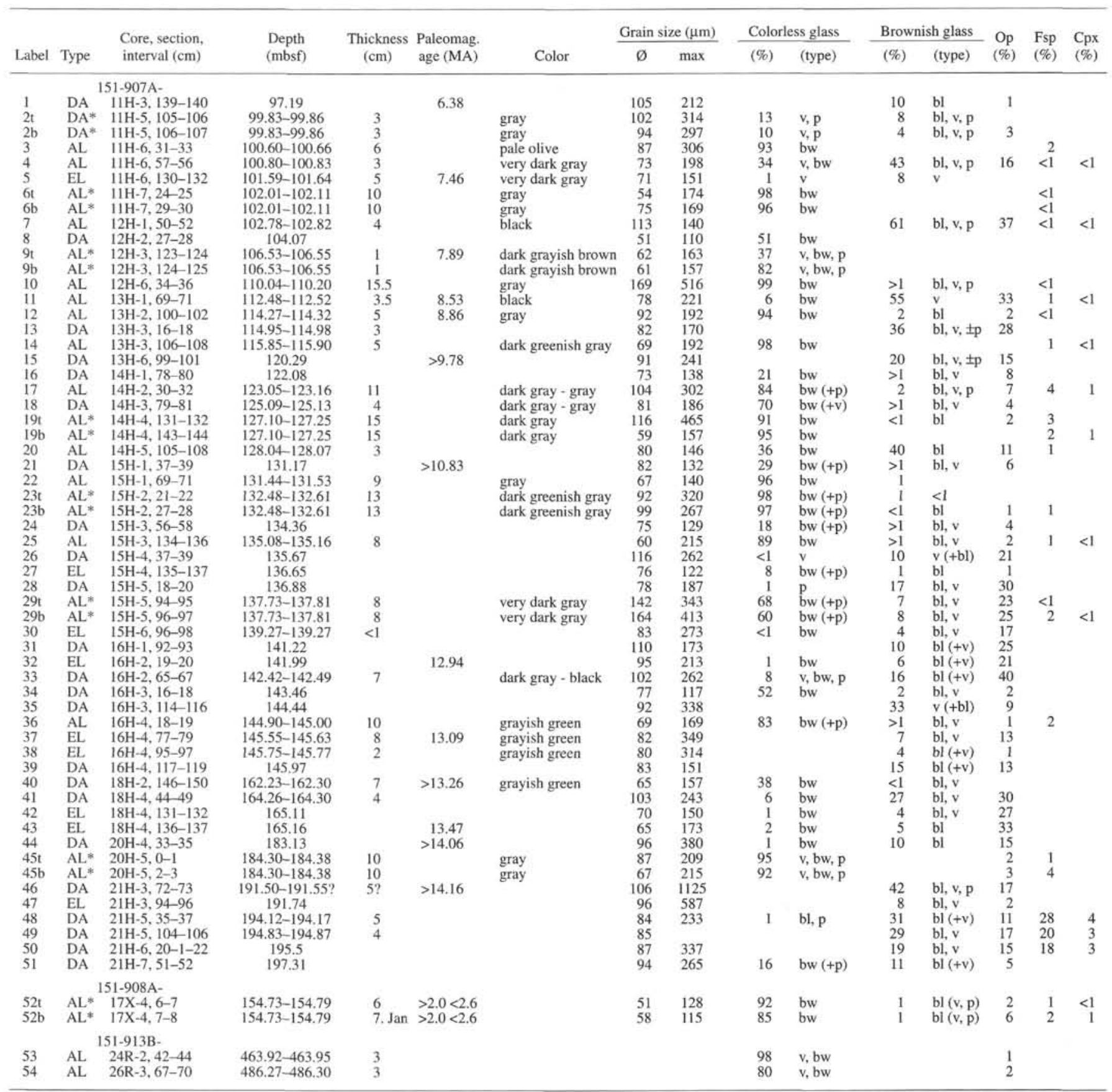

Notes: With a few exceptions, depth intervals, thickness, paleomagnetic ages and colors are taken from Shipboard Scientific Party $(1995 \mathrm{a}-\mathrm{c})$. Type: DA $=$ disseminated ash layer; EL $=$ ash-enriched ash layer; $\mathrm{AL}=$ discrete ash layer; *layer sampled at two depth intervals. Grain size: $\emptyset=$ average grain size; max = maximum grain size (for explanation see methods chapter). Components: $\%=$ modal percentage (see methods chapter, this study); $v=$ vesicular shards, $b w=$ bubble wall shards, $\mathrm{p}=$ pumice fragments, $\mathrm{bl}=\mathrm{blocky}$ shards; $( \pm \mathrm{p})$ $=$ sample contains subordinate pumice fragments; $\mathrm{Op}=$ tachylite and opaques, Fsp = feldspar; Cpx = clinopyroxene.

VG-2, 864-M1-05, 89/15GLBX) were analyzed periodically to monitor the accuracy of the microprobe. The standard SCAPOLITE was run for sulfur analyses. Sulfur concentrations of $1010 \pm 70 \mathrm{ppm}$ obtained for the natural glass standard ALV-981R23-5 (submarine basalt) correspond to the values of $1050 \pm 140$ ppm reported by Metrich et al. (1991) and $1070 \pm 90 \mathrm{ppm}$ reported by Metrich and Clocchiatti
(1989). The precision of the sulfur analyses is estimated to be \pm 80 $\mathrm{ppm}$ at the $1000 \mathrm{ppm}$ sulfur level.

In order to effectively average compositional heterogeneities, up to 8 points were analyzed on each glass particle. The data presented are averages of these analyses. Altogether up to 30 individual glass particles were analyzed for each ash deposit (Tables 3,4 ). 
Table 2. Comparison of major element analyses of the homogeneous LIPARI obsidian.

\begin{tabular}{lrrrrr}
\hline & \multicolumn{5}{c}{ LIPARI } \\
\cline { 2 - 6 } & $\begin{array}{c}\text { XRF } \\
\text { (Sparks, pers. } \\
\text { comm., 1990) }\end{array}$ & \multicolumn{2}{c}{ EMP } & \multicolumn{2}{c}{ EMP } \\
& (Hunt \& Hill 1993) & \multicolumn{2}{c}{ (this study) } \\
\hline & & & $\mathrm{S}$ (n-1) & $\mathrm{n}=30$ & St. dev. \\
$\mathrm{SiO}_{2}$ & 74.03 & 74.35 & 0.17 & 73.94 & 0.20 \\
$\mathrm{TiO}_{2}$ & 0.08 & & & 0.09 & 0.03 \\
$\mathrm{Al}_{2} \mathrm{O}_{3}$ & 12.72 & 12.87 & 0.07 & 12.95 & 0.07 \\
$\mathrm{Fe}_{2} \mathrm{O}_{3}$ & 1.73 & & & & \\
$\mathrm{FeO}^{*}$ & & 1.51 & 0.05 & 1.50 & 0.07 \\
$\mathrm{MnO}_{\mathrm{MgO}}$ & 0.08 & 0.07 & 0.02 & 0.07 & 0.04 \\
$\mathrm{CaO}$ & 0.00 & 0.05 & 0.01 & 0.05 & 0.02 \\
$\mathrm{Na}{ }_{2} \mathrm{O}$ & 0.72 & 0.74 & 0.02 & 0.73 & 0.04 \\
$\mathrm{~K}_{2} \mathrm{O}$ & 4.06 & 3.93 & 0.06 & 4.25 & 0.04 \\
$\mathrm{LOI}$ & 5.18 & 5.11 & 0.04 & 5.10 & 0.07 \\
$\mathrm{Total}$ & 1.04 & & & & \\
\hline
\end{tabular}

Note: Analysis performed using an X-ray fluorescence (XRF) spectrometer (Sparks, pers. comm., 1990), a Cameca Camebax 4 electron microprobe (Hunt and Hill, 1993), and a Cameca SX 50 electron microprobe (this study).

\section{LITHOLOGY, STRATIGRAPHY AND STRUCTURE}

Volcanic glass occurs as light to dark brown, „opaque (tachylitic), and colorless fragments in ash horizons with varying contents of glass particles from approximately $3 \%$ to $99 \%$. Three types of ash deposits can be distinguished based on structure and modal compositions: (1) discrete ash layers $=>80 \%$ glass particles, sharp base and gradational top (AL in Table 1); (2) disseminated ash layers $=10 \%-$ $80 \%$ glass particles (DA in Table 1); and (3) ash-enriched layers $=$ deposits with $5 \%-10 \%$ volcanic particles (EL in Table 1). Altogether 18 discrete ash layers, 24 disseminated ash layers, and 9 ash-enriched layers were identified in the Miocene part of Hole 907A; the two ash deposits in Hole 913B and the one in Hole 908A are discrete ash layers.

Ash deposits are preserved throughout Cores 151-907A-11H to $21 \mathrm{H}$, with the exception of Cores $151-907 \mathrm{~A}-17 \mathrm{H}$ and $19 \mathrm{H}$ (Fig. 2), over a depth interval of $100 \mathrm{~m}$ (97.19-197.31 meters below sea level (mbsf)). This interval corresponds to a paleomagnetic age of $6.4 \mathrm{Ma}$ (Chron C3An.2n) to 14 Ma (Shipboard Scientific Party, 1995a). In the late Miocene part of this sequence (97.19-128.07 mbsf, Cores $151-907 \mathrm{~A}-11 \mathrm{H}$ to $14 \mathrm{H})$, the distribution of ash deposits is regular with an average of five layers per core or of one per million years according to sedimentation rates (Shipboard Scientific Party, 1995a). The abundance of ash deposits rises abruptly on top of the middle Miocene sequence of Hole 907A. Nineteen ash deposits were found in Cores 151-907A-15H and $16 \mathrm{H}$ over an interval of $<15 \mathrm{~m}$ (131.17$145.97 \mathrm{mbsf}$ ). Ash deposits occur more sporadically further downhole in Cores 151-907A-18H (4 ash deposits) and 20H ( 2 ash deposits). In the lower part of Core 151-907A-21H, the number of ash deposits increases again significantly to 6 between 191.50 and 197.31 mbsf. Ash deposits are absent below $197.40 \mathrm{mbsf}$. The abundance of discrete ash layers decreases continuously downhole, in contrast to the total amount of ash deposits. The ratio of discrete over disseminated ash layers amounts to $1: 1$ in the late Miocene sequence, but it is $1: 2$ on top of the middle Miocene sequence (Cores $151-907 \mathrm{~A}-15 \mathrm{H}$ and $16 \mathrm{H})$ and decreases to $1: 8$ further downhole.

The thickness of discrete ash layers varies from 1 to $15.5 \mathrm{~cm}$, but some are heavily burrowed, which makes precise measurement difficult. The thickness of most disseminated and ash-enriched layers cannot be determined because of the lack of distinct contacts to the sediments above and below. Some layers extend obviously over some tens of centimeters ("ash zones"). All discrete and some dis- seminated ash layers can be easily identified by their colors, which range from pale olive, greenish gray, and gray to black (Munsell color card; Shipboard Scientific Party, 1995a), whereas the color of most disseminated ash layers and all ash-enriched layers do not differ from the surrounding sediments.

The darkness of the ash deposits depends on the amount of brownish glass particles and opaque components. Color and thus inferred composition of the studied ash deposits correlate possibly with their thickness in contrast to observations on Cenozoic ash layers from the Vøring Plateau (Bitschene et al., 1989). All discrete ash layers thicker than $5 \mathrm{~cm}$ are gray, greenish gray, dark gray, or pale olive and consist predominantly of colorless shards, whereas discrete ash layers, disseminated ash layers of visible thickness containing predominantly brownish glass fragments, or ash layers of bimodal composition are very dark gray to black and thinner than $5 \mathrm{~cm}$ (Table 1).

\section{TEXTURE AND COMPOSITION}

The average medium diameter of the glass particles in Leg 151 ash deposits is approximately $100 \pm 60 \mu \mathrm{m}$ (coarse silt to very fine sand). These values match those published by Lacasse et al. (this volume) for the Pliocene and Pleistocene sequence of Hole 907A and Bitschene et al. (1989) for ash deposits of the Vøring Plateau. Maximum grain sizes of glass particle ( 5 largest particles) vary strongly from 150 to $1125 \mu \mathrm{m}$ (Table 1). All ash deposits consisting mainly of colorless glass particles are well sorted; sorting of bimodal deposits and those consisting mainly of brownish particles is poor to good.

Colorless glass particles dominate in about $45 \%$ of the ash deposits recovered in the Miocene part of Hole 907A, brownish shards in almost $50 \%$, and more than $5 \%$ are bimodal (Table 1). Whereas almost $70 \%$ of the ash deposits in the upper part of this sequence (Cores $151-907 \mathrm{~A}-11 \mathrm{H}$ to $15 \mathrm{H}$ ) are dominated by colorless glass, more than $75 \%$ of the ash deposits in the lower part (Cores 151-907A-16 H to $21 \mathrm{H}$ ) contain mainly brownish particles. All ash layers in Holes $908 \mathrm{~A}$ and $913 \mathrm{~B}$ and 13 ash layers from Hole $907 \mathrm{~A}$ consist of $80 \%-99 \%$ colorless glass shards and therefore are clearly felsic. The other extreme are mafic deposits containing 30\%-60\% brownish glass particles (sideromelane) and up to $40 \%$ tachylite. All transitions exist between these two endmembers. We restrict the term bimodal to ash layers and disseminated ash layers with ratios of silicic over brownish glass particles and tachylite between $6: 4$ and 4:6.

The majority of the colorless glass particles are platy or cuspate bubble wall shards, tabular pumice with parallel alignment of elongated vesicles occur in almost $60 \%$ of the silicic to bimodal layers (particularly in layers 2, 9, 33, and 45; Table 1). In 25\% of these layers vesicular to highly vesicular or pumice-like shards with round bubbles occur in various amounts. Dense blocky to slightly vesicular shards are most common in almost $80 \%$ of the brownish glass populations; although, among the light brown glass particles, vesicular shards dominate over tabular pumice and blocky fragments. Dark brown shards occur mostly as dense blocky shards or with a few spherical vesicles; vesicular or pumice-like dark brown shards are rare. Additional glass phases are opaque tachylite and altered and/or hydrated glass.

Colorless glass particles are felsic, whereas almost all brownish glass particles are mafic to intermediate. However, some light brown glass particles occurring in felsic ash deposits may have also a felsic composition. In general the darkness of the brown glass appears to depend on the $\mathrm{TiO}_{2}$ and $\mathrm{FeO}$ (total iron calculated as $\mathrm{FeO}^{*}$ ) content of the glass. Dark brown glass particles usually have high and light brown particles low $\mathrm{TiO}_{2}$ and $\mathrm{FeO}^{*}$ concentrations.

Major additional constituents of the ash deposits are opaque components and various amounts of lithic clasts, organic material, and clay. Crystals are rare (usually $<3$ mod.\%) except in the disseminated layers 47,48 , and 50 in Hole $907 \mathrm{~A}(18 \%-20 \%$ feldspar and $3 \%-4 \%$ 
Table 3. Electron microprobe analyses of silicic glassy tephra recovered at Holes 907A (Miocene sequence), 908A, and 913B.

\begin{tabular}{|c|c|c|c|c|c|c|c|c|c|c|}
\hline Sample: & \multicolumn{2}{|c|}{$151-907 \mathrm{~A}-11 \mathrm{H}-5$} & \multicolumn{2}{|c|}{$151-907 \mathrm{~A}-11 \mathrm{H}-5$} & \multicolumn{2}{|c|}{$151-907 \mathrm{~A}-11 \mathrm{H}-6$} & \multicolumn{2}{|c|}{$151-907 \mathrm{~A}-11 \mathrm{H}-6$} & \multicolumn{2}{|c|}{$151-907 \mathrm{~A}-11 \mathrm{H}-6$} \\
\hline Interval $(\mathrm{cm})$ : & \multicolumn{2}{|c|}{$105-106$} & \multicolumn{2}{|c|}{$105-106$} & \multicolumn{2}{|c|}{$31-33$} & \multicolumn{2}{|c|}{$56-57$} & \multicolumn{2}{|c|}{$24-25,29-30$} \\
\hline Label: & \multicolumn{2}{|c|}{2 (pop. 1 ) } & \multicolumn{2}{|c|}{2 (pop. II) } & \multicolumn{2}{|c|}{3} & \multicolumn{2}{|c|}{4} & \multicolumn{2}{|c|}{6 (pop. I) } \\
\hline Type: & \multicolumn{2}{|c|}{$\mathrm{DA}$} & \multicolumn{2}{|c|}{ DA } & \multicolumn{2}{|c|}{$\mathrm{AL}$} & \multicolumn{2}{|c|}{$\mathrm{AL}$} & \multicolumn{2}{|c|}{ AL } \\
\hline Particle/points: & \multicolumn{2}{|c|}{$5 \mathrm{f} / 10 \mathrm{p}$} & \multicolumn{2}{|c|}{$5 f / 10 p$} & \multicolumn{2}{|c|}{$8 f / 16 p$} & \multicolumn{2}{|c|}{$7 f / 13 p$} & \multicolumn{2}{|c|}{$7 f / 17 p$} \\
\hline & Average & St. dev. & Average & St. dev. & Average & St. dev. & Average & St. dev. & Average & St. dev. \\
\hline $\begin{array}{l}\mathrm{SiO}_{2} \\
\mathrm{TiO}_{2} \\
\mathrm{Al}_{2} \mathrm{O}_{3} \\
\mathrm{FeO}^{*} \\
\mathrm{MnO} \\
\mathrm{MgO} \\
\mathrm{CaO} \\
\mathrm{Na}_{2} \mathrm{O} \\
\mathrm{K}_{2} \mathrm{O}\end{array}$ & $\begin{array}{r}71.06 \\
0.30 \\
12.33 \\
2.54 \\
0.08 \\
0.09 \\
1.49 \\
4.00 \\
2.68\end{array}$ & $\begin{array}{l}0.70 \\
0.13 \\
0.60 \\
0.46 \\
0.04 \\
0.06 \\
0.10 \\
0.09 \\
0.17\end{array}$ & $\begin{array}{r}73.72 \\
0.07 \\
11.92 \\
1.03 \\
0.02 \\
0.03 \\
0.95 \\
3.52 \\
3.18\end{array}$ & $\begin{array}{l}0.71 \\
0.05 \\
0.29 \\
0.04 \\
0.01 \\
0.01 \\
0.07 \\
0.20 \\
0.24\end{array}$ & $\begin{array}{r}68.43 \\
0.28 \\
12.98 \\
2.43 \\
0.08 \\
0.24 \\
1.29 \\
4.73 \\
3.00\end{array}$ & $\begin{array}{l}0.45 \\
0.04 \\
0.10 \\
0.16 \\
0.03 \\
0.04 \\
0.09 \\
0.06 \\
0.08\end{array}$ & $\begin{array}{r}69.00 \\
0.29 \\
12.38 \\
4.13 \\
0.15 \\
0.02 \\
1.97 \\
4.57 \\
2.15\end{array}$ & $\begin{array}{l}0.63 \\
0.06 \\
0.20 \\
0.28 \\
0.04 \\
0.03 \\
0.11 \\
0.08 \\
0.06\end{array}$ & $\begin{array}{r}72.35 \\
0.15 \\
12.24 \\
1.81 \\
0.05 \\
0.05 \\
0.75 \\
4.31 \\
3.11\end{array}$ & $\begin{array}{l}0.97 \\
0.04 \\
0.27 \\
0.04 \\
0.02 \\
0.03 \\
0.04 \\
0.17 \\
0.13\end{array}$ \\
\hline Total & 94.56 & & 94.44 & & 93.47 & & 94.67 & & 94.82 & \\
\hline Sample: & \multicolumn{2}{|c|}{$151-907 \mathrm{~A}-11 \mathrm{H}-6$} & \multicolumn{2}{|c|}{$151-907 \mathrm{~A}-12 \mathrm{H}-2$} & \multicolumn{2}{|c|}{$151-907 \mathrm{~A}-12 \mathrm{H}-3$} & \multicolumn{2}{|c|}{$151-907 \mathrm{~A}-12 \mathrm{H}-3$} & $151-907$ & $-12 \mathrm{H}-6$ \\
\hline Interval $(\mathrm{cm})$ : & $24-25$ & $9-30$ & 27 & & $123-124$ & $24-125$ & $123-12$ & $124-125$ & & \\
\hline Label: & $6(p$ & II) & 8 & & $9(\mathrm{p}$ & l) & $9(\mathrm{p}$ & II) & 1 & \\
\hline Type: & & & D. & & & & & & & \\
\hline Particle/points: & & & $8 \mathrm{f} / 1$ & & & & & & 9f/ & \\
\hline $\begin{array}{l}\mathrm{SiO}_{2} \\
\mathrm{TiO}_{2} \\
\mathrm{Al}_{2} \mathrm{O}_{3} \\
\mathrm{FeO}^{*} \\
\mathrm{MnO} \\
\mathrm{MgO} \\
\mathrm{CaO} \\
\mathrm{Na}_{2} \mathrm{O} \\
\mathrm{K}_{2} \mathrm{O}\end{array}$ & $\begin{array}{c}\text { Average } \\
70.98 \\
0.19 \\
11.98 \\
2.50 \\
0.10 \\
0.04 \\
1.05 \\
4.51 \\
2.68\end{array}$ & $\begin{array}{c}\text { St. dev. } \\
0.92 \\
0.04 \\
0.17 \\
0.17 \\
0.03 \\
0.03 \\
0.09 \\
0.13 \\
0.10\end{array}$ & $\begin{array}{r}\text { Average } \\
71.28 \\
0.11 \\
12.15 \\
1.68 \\
0.04 \\
0.13 \\
1.21 \\
4.43 \\
2.50\end{array}$ & $\begin{array}{c}\text { St. dev. } \\
0.30 \\
0.07 \\
0.17 \\
0.10 \\
0.03 \\
0.02 \\
0.09 \\
0.11 \\
0.12\end{array}$ & $\begin{array}{c}\text { Average } \\
60.60 \\
0.47 \\
15.41 \\
5.17 \\
0.22 \\
0.08 \\
1.49 \\
6.00 \\
4.69\end{array}$ & $\begin{array}{c}\text { St. dev. } \\
0.34 \\
0.08 \\
0.35 \\
0.33 \\
0.02 \\
0.04 \\
0.10 \\
0.18 \\
0.09\end{array}$ & $\begin{array}{c}\text { Average } \\
61.72 \\
0.25 \\
15.17 \\
4.61 \\
0.22 \\
0.01 \\
1.05 \\
6.43 \\
4.50\end{array}$ & $\begin{array}{c}\text { St. dev. } \\
0.22 \\
0.07 \\
0.27 \\
0.25 \\
0.04 \\
0.01 \\
0.04 \\
0.10 \\
0.05\end{array}$ & $\begin{array}{r}\text { Average } \\
70.77 \\
0.14 \\
11.71 \\
2.14 \\
0.10 \\
0.02 \\
0.85 \\
4.43 \\
3.22\end{array}$ & $\begin{array}{c}\text { St. dev. } \\
0.30 \\
0.03 \\
0.06 \\
0.06 \\
0.01 \\
0.01 \\
0.03 \\
0.10 \\
0.07\end{array}$ \\
\hline Total & 94.04 & & 93.55 & & 94.13 & & 93.97 & & 93.37 & \\
\hline Sample: & $151-907$ & $131 \mathrm{H}-1$ & $151-907$ & $-13 \mathrm{H}-2$ & $151-907$ & $-13 \mathrm{H}-3$ & $151-90^{-}$ & $-14 \mathrm{H}-2$ & $151-907$ & $-14 \mathrm{H}-3$ \\
\hline Interval $(\mathrm{cm})$ : & & & 100 & 102 & 106 & & & & & 81 \\
\hline Label: & & & 1 & & & & & & 1 & \\
\hline Type: & & & A & & & & & & & \\
\hline Particle/points: & & & $6 \mathrm{f} / 1$ & & & & & & & \\
\hline $\begin{array}{l}\mathrm{SiO}_{2} \\
\mathrm{TiO}_{2} \\
\mathrm{Al}_{2} \mathrm{O}_{3} \\
\mathrm{FeO}^{*} \\
\mathrm{MnO} \\
\mathrm{MgO} \\
\mathrm{CaO} \\
\mathrm{Na}_{2} \mathrm{O} \\
\mathrm{K}_{2} \mathrm{O}\end{array}$ & $\begin{array}{c}\text { Average } \\
70.31 \\
0.28 \\
12.25 \\
3.18 \\
0.14 \\
0.04 \\
1.28 \\
4.18 \\
2.73\end{array}$ & $\begin{array}{c}\text { St. dev. } \\
0.60 \\
0.07 \\
0.07 \\
0.29 \\
0.01 \\
0.02 \\
0.14 \\
0.23 \\
0.20\end{array}$ & $\begin{array}{r}\text { Average } \\
70.77 \\
0.20 \\
11.59 \\
2.20 \\
0.08 \\
0.01 \\
1.08 \\
4.30 \\
2.82\end{array}$ & $\begin{array}{c}\text { St. dev. } \\
0.45 \\
0.05 \\
0.06 \\
0.04 \\
0.03 \\
0.01 \\
0.01 \\
0.12 \\
0.06\end{array}$ & $\begin{array}{c}\text { Average } \\
70.24 \\
0.33 \\
11.95 \\
4.13 \\
0.16 \\
0.02 \\
1.63 \\
4.47 \\
2.27\end{array}$ & $\begin{array}{c}\text { St. dev. } \\
0.42 \\
0.04 \\
0.09 \\
0.12 \\
0.02 \\
0.01 \\
0.07 \\
0.12 \\
0.10\end{array}$ & $\begin{array}{c}\text { Average } \\
71.47 \\
0.15 \\
12.42 \\
2.34 \\
0.08 \\
0.02 \\
1.17 \\
4.28 \\
2.87\end{array}$ & $\begin{array}{c}\text { St. dev. } \\
0.31 \\
0.07 \\
0.11 \\
0.11 \\
0.02 \\
0.01 \\
0.08 \\
0.17 \\
0.31\end{array}$ & $\begin{array}{r}\text { Average } \\
69.69 \\
0.31 \\
12.65 \\
3.17 \\
0.15 \\
0.08 \\
0.98 \\
4.90 \\
2.68\end{array}$ & $\begin{array}{c}\text { St. dev. } \\
0.27 \\
0.08 \\
0.06 \\
0.11 \\
0.02 \\
0.02 \\
0.03 \\
0.09 \\
0.06\end{array}$ \\
\hline Total & 94.38 & & 93.05 & & 95.19 & & 94.80 & & 94.61 & \\
\hline Sample: & $151-90$ & $-14 \mathrm{H}-4$ & $151-907$ & $-15 \mathrm{H}-1$ & $151-907$ & $-15 \mathrm{H}-2$ & $151-90$ & $-15 \mathrm{H}-3$ & $151-907$ & $-15 \mathrm{H}-5$ \\
\hline Interval $(\mathrm{cm})$ : & $131-132$ & $43-144$ & 69 & & $21-22$ & $7-28$ & 134 & 136 & 94 & 95 \\
\hline Label: & & & 22 & & & & & & & \\
\hline Type: & & & Al & & & & & & AL & op) \\
\hline Particle/points: & & & $10 \mathrm{f} /$ & $1 p$ & & & & & $6 \mathrm{f} /$ & \\
\hline & Average & St. dev. & Average & St. dev. & Average & St. dev. & Average & St. dev. & Average & St. dev. \\
\hline $\begin{array}{l}\mathrm{SiO}_{2} \\
\mathrm{TiO}_{2} \\
\mathrm{Al}_{2} \mathrm{O}_{3} \\
\mathrm{FeO}^{*} \\
\mathrm{MnO} \\
\mathrm{MgO} \\
\mathrm{CaO} \\
\mathrm{Na}_{2} \mathrm{O} \\
\mathrm{K}_{2} \mathrm{O}\end{array}$ & $\begin{array}{r}70.89 \\
0.15 \\
12.48 \\
2.53 \\
0.10 \\
0.00 \\
1.08 \\
4.39 \\
3.07\end{array}$ & $\begin{array}{l}0.62 \\
0.04 \\
0.10 \\
0.15 \\
0.03 \\
0.00 \\
0.09 \\
0.07 \\
0.11\end{array}$ & $\begin{array}{r}69.47 \\
0.22 \\
12.72 \\
3.48 \\
0.12 \\
0.08 \\
1.49 \\
4.46 \\
2.44\end{array}$ & $\begin{array}{l}0.32 \\
0.03 \\
0.06 \\
0.12 \\
0.03 \\
0.02 \\
0.04 \\
0.07 \\
0.07\end{array}$ & $\begin{array}{r}73.29 \\
0.17 \\
11.73 \\
2.10 \\
0.07 \\
0.03 \\
0.86 \\
4.25 \\
2.56\end{array}$ & $\begin{array}{l}0.55 \\
0.05 \\
0.15 \\
0.10 \\
0.03 \\
0.01 \\
0.07 \\
0.12 \\
0.15\end{array}$ & $\begin{array}{r}72.59 \\
0.20 \\
11.65 \\
2.75 \\
0.10 \\
0.00 \\
1.11 \\
4.49 \\
2.35\end{array}$ & $\begin{array}{l}0.34 \\
0.04 \\
0.04 \\
0.20 \\
0.04 \\
0.01 \\
0.07 \\
0.06 \\
0.11\end{array}$ & $\begin{array}{r}72.21 \\
0.32 \\
11.45 \\
2.65 \\
0.10 \\
0.10 \\
1.48 \\
4.21 \\
2.00\end{array}$ & $\begin{array}{l}0.39 \\
0.06 \\
0.05 \\
0.23 \\
0.03 \\
0.03 \\
0.05 \\
0.13 \\
0.10\end{array}$ \\
\hline Total & 94.69 & & 94.47 & & 95.06 & & 95.23 & & 94.52 & \\
\hline
\end{tabular}


Table 3 (continued).

\begin{tabular}{|c|c|c|c|c|c|c|c|c|c|c|}
\hline Sample: & \multicolumn{2}{|c|}{ 151-907A-15H-5 } & \multicolumn{2}{|c|}{$151-907 \mathrm{~A}-16 \mathrm{H}-2$} & \multicolumn{2}{|c|}{$151-907 \mathrm{~A}-16 \mathrm{H}-4$} & \multicolumn{2}{|c|}{$151-907 \mathrm{~A}-18 \mathrm{H}-2$} & \multicolumn{2}{|c|}{$151-907 \mathrm{~A}-20 \mathrm{H}-5$} \\
\hline Interval $(\mathrm{cm})$ : & \multicolumn{2}{|c|}{ 96-97 } & \multicolumn{2}{|c|}{$65-67$} & \multicolumn{2}{|c|}{$18-19$} & \multicolumn{2}{|c|}{$146-150$} & \multicolumn{2}{|c|}{$0-1,2-3$} \\
\hline Label: & \multicolumn{2}{|c|}{$29 \mathrm{t}$} & \multicolumn{2}{|c|}{33} & \multicolumn{2}{|c|}{36} & \multicolumn{2}{|c|}{40} & \multicolumn{2}{|c|}{45} \\
\hline Type: & \multicolumn{2}{|c|}{ AL (base) } & \multicolumn{2}{|c|}{ (DA) } & \multicolumn{2}{|c|}{ AL } & \multicolumn{2}{|c|}{$\mathrm{DA}$} & \multicolumn{2}{|c|}{$\mathrm{AL}$} \\
\hline Particle/points: & \multicolumn{2}{|c|}{$9 f / 24 p$} & \multicolumn{2}{|c|}{$12 f / 25 p$} & \multicolumn{2}{|c|}{$11 \mathrm{f} / 24 \mathrm{p}$} & \multicolumn{2}{|c|}{$12 f / 15 p$} & \multicolumn{2}{|c|}{$28 \mathrm{f} / 74 \mathrm{p}$} \\
\hline & Average & St. dev. & Average & St. dev. & Average & St. dev. & Average & St. dev. & Average & St. dev. \\
\hline $\begin{array}{l}\mathrm{SiO}_{2} \\
\mathrm{TiO}_{2} \\
\mathrm{Al}_{2} \mathrm{O}_{3} \\
\mathrm{FeO}^{*} \\
\mathrm{MnO} \\
\mathrm{MgO} \\
\mathrm{CaO} \\
\mathrm{Na}_{2} \mathrm{O} \\
\mathrm{K}_{2} \mathrm{O}\end{array}$ & $\begin{array}{r}70.50 \\
0.30 \\
11.39 \\
2.64 \\
0.09 \\
0.12 \\
1.50 \\
4.37 \\
2.03\end{array}$ & $\begin{array}{l}0.33 \\
0.06 \\
0.04 \\
0.04 \\
0.02 \\
0.01 \\
0.05 \\
0.09 \\
0.06\end{array}$ & $\begin{array}{r}71.31 \\
0.11 \\
12.31 \\
1.80 \\
0.07 \\
0.03 \\
1.26 \\
4.46 \\
2.33\end{array}$ & $\begin{array}{l}0.27 \\
0.04 \\
0.06 \\
0.06 \\
0.02 \\
0.01 \\
0.03 \\
0.08 \\
0.15\end{array}$ & $\begin{array}{r}67.86 \\
0.38 \\
12.41 \\
4.62 \\
0.12 \\
0.00 \\
1.88 \\
4.20 \\
3.11\end{array}$ & $\begin{array}{l}0.52 \\
0.05 \\
0.30 \\
0.22 \\
0.02 \\
0.00 \\
0.15 \\
0.12 \\
0.07\end{array}$ & $\begin{array}{r}70.27 \\
0.19 \\
11.66 \\
2.90 \\
0.09 \\
0.04 \\
1.21 \\
4.42 \\
2.74\end{array}$ & $\begin{array}{l}0.32 \\
0.06 \\
0.07 \\
0.11 \\
0.03 \\
0.01 \\
0.04 \\
0.17 \\
0.19\end{array}$ & $\begin{array}{r}73.01 \\
0.18 \\
11.53 \\
1.38 \\
0.04 \\
0.06 \\
0.61 \\
3.74 \\
3.97\end{array}$ & $\begin{array}{l}0.38 \\
0.03 \\
0.14 \\
0.09 \\
0.02 \\
0.01 \\
0.06 \\
0.15 \\
0.25\end{array}$ \\
\hline Total & \multicolumn{2}{|l|}{92.93} & \multicolumn{2}{|l|}{93.68} & \multicolumn{2}{|l|}{94.57} & \multicolumn{2}{|l|}{93.52} & \multicolumn{2}{|l|}{94.52} \\
\hline Sample: & \multicolumn{2}{|c|}{$151-907 \mathrm{~A}-21 \mathrm{H}-5$} & \multicolumn{2}{|c|}{$151-907 \mathrm{~A}-21 \mathrm{H}-7$} & \multicolumn{2}{|c|}{$151-908 \mathrm{~A}-17 \mathrm{X}-5$} & \multicolumn{2}{|c|}{$151-913 \mathrm{~B}-24 \mathrm{R}-2$} & & \\
\hline Interval $(\mathrm{cm})$ : & \multicolumn{2}{|c|}{$35-37$} & \multicolumn{2}{|c|}{$51-52$} & 6-7 & $7-8$ & & & & \\
\hline Label: & & & 5 & & & & & & & \\
\hline Type: & & & D & & & & & & & \\
\hline Particle/points: & & & $8 \mathrm{f} /$ & $0 p$ & & & & & & \\
\hline & Average & St. dev. & Average & St. dev. & Average & St. dev. & Average & St. dev. & & \\
\hline $\begin{array}{l}\mathrm{SiO}_{2} \\
\mathrm{TiO}_{2} \\
\mathrm{Al}_{2} \mathrm{O}_{3} \\
\mathrm{FeO}^{*} \\
\mathrm{MnO} \\
\mathrm{MgO} \\
\mathrm{CaO} \\
\mathrm{Na}_{2} \mathrm{O} \\
\mathrm{K}_{2} \mathrm{O}\end{array}$ & $\begin{array}{r}71.19 \\
0.18 \\
11.46 \\
2.34 \\
0.10 \\
0.02 \\
1.08 \\
3.94 \\
3.44\end{array}$ & $\begin{array}{l}0.41 \\
0.04 \\
0.15 \\
0.03 \\
0.03 \\
0.02 \\
0.05 \\
0.30 \\
0.15\end{array}$ & $\begin{array}{r}69.31 \\
0.37 \\
11.81 \\
3.74 \\
0.12 \\
0.17 \\
2.05 \\
4.67 \\
2.70\end{array}$ & $\begin{array}{l}0.64 \\
0.07 \\
0.15 \\
0.27 \\
0.07 \\
0.06 \\
0.22 \\
0.12 \\
0.08\end{array}$ & $\begin{array}{r}70.92 \\
0.15 \\
12.25 \\
2.33 \\
0.09 \\
0.00 \\
1.07 \\
4.78 \\
2.32\end{array}$ & $\begin{array}{l}0.75 \\
0.04 \\
0.28 \\
0.17 \\
0.03 \\
0.00 \\
0.06 \\
0.13 \\
0.15\end{array}$ & $\begin{array}{r}66.24 \\
0.21 \\
13.11 \\
3.13 \\
0.15 \\
0.01 \\
0.78 \\
4.22 \\
4.70\end{array}$ & $\begin{array}{l}0.36 \\
0.06 \\
0.11 \\
0.14 \\
0.05 \\
0.01 \\
0.04 \\
0.30 \\
0.13\end{array}$ & & \\
\hline Total & 93.75 & & 94.96 & & 93.90 & & 92.55 & & & \\
\hline
\end{tabular}

Note: $\mathrm{AL}=$ discrete ash layer, $\mathrm{DA}=$ disseminated ash layer, $\mathrm{EL}=$ ash-enriched layer; type in parentheses $(\mathrm{AL})=$ subordinate silicic population in a mafic or intermediate layer; particle/points $=$ number of analyzed particles ( $f$ ) and total number of analyses (p).

clinopyroxene, Table 1). Feldspar and clinopyroxene are the dominant mineral phases; other minerals, such as detritic quartz, olivine, amphibole, biotite, opaques (magnetite, pyrite), and diagenetic minerals (e.g., glauconite), are minor. The minerals vary widely in grain size, and only a few are euhedral. Most are broken and occur as small crystal fragments $(<200 \mu \mathrm{m})$. There is no clear evidence if these crystals and crystal fragments, respectively, are phenocrysts, xenocrysts, or epiclastic particles. Only the coexistence of feldspar (plagioclase) and clinopyroxene with brownish glass particles and tachylite in some ash deposits suggest that these crystals are phenocrysts.

\section{MAJOR ELEMENT COMPOSITION}

Thirty-four ash deposits from the Miocene sequence of Hole 907A and one each from Hole 908A and 913B were selected for chemical analyses (Tables 3,4). The totals of microprobe analyses of mafic and intermediate glass particles range from 98.5 to $100.5 \mathrm{wt} \%$, of felsic glass from 92.5 to $95.5 \mathrm{wt} \%$ (with iron calculated as $\mathrm{FeO}$ *). The latter is essentially due to hydration of silicic glasses and/or high initial volatile contents in felsic magmas ranging, for example, from 2 to 7 wt $\% \mathrm{H}_{2} \mathrm{O}$ (e.g., Bitschene and Schmincke, 1990).

The analyzed glass shards comprise basalts, basaltic andesites, andesites, dacites, trachytes, and rhyolites (Fig. 3). Whereas the Miocene tephra (Holes 907A and 908A) plot basically in the same fields, the late Eocene to early Oligocene tephra recovered in Hole 913B clearly differ in composition. All rhyolitic tephra have an Icelandic affinity, whereas the trachytic ash layer (Sample 151-907A$12 \mathrm{H}-3,122-125 \mathrm{~cm}$ ) plots within the Jan Mayen field (Fig. 3). Most basaltic to dacitic tephra match the Icelandic rock series, and some basalts with low silica content also match the Jan Mayen rock series, but a part of the mafic and intermediate tephra show somewhat lower alkali content than does the Icelandic series. For these reasons and because of the high age (ash layer in Hole 913B) and long distance (e.g., Site 908) from Iceland, additional sources for the analyzed tephra must be taken into account.

Almost $45 \%$ of the analyzed ash deposits contain only rhyolitic glass particles (Table 3 ), $26 \%$ only mafic and/or intermediate particles (Table 4 ), and $26 \%$ both mafic to intermediate and rhyolitic shards in significant amounts (Tables 3, 4). Only one ash deposit (no. 9 in Core $151-907 \mathrm{~A}-12 \mathrm{H}$ ) is trachytic in composition with two different compositional populations (e.g., Fig. 3). The composition of the rhyolitic shards is mostly homogeneous within an individual deposit, only three layers (nos. 2, 6, and 29) show two different compositional populations of rhyolitic shards; the shards in the upper part of the layer are slightly more evolved in one of them than the shards in the lower part (layer 29 in Core 151-907A-15H). Almost $40 \%$ of the deposits containing mafic to intermediate particles show clear trends from basaltic to andesitic or dacitic endmembers (layers 2, 4, 7, 11, 17, 29, and 52). Within the remaining deposits, basaltic particles are also compositionally homogeneous within a single layer, except for layers 17,29 , and 36 . The top of layer 29 is more evolved (basaltic to andesitic) than the base (homogeneous basaltic).

Analyses of three individual ash deposits, shown in Table 5, were made with both microprobe systems at GEOMAR (Kiel, Germany) and Brown University (Rhode Island, U.S.A.) to make our chemical analyses comparable with those of the Hole 907A Pliocene and Pleistocene ash deposits presented by Lacasse et al. (this volume). Except for $\mathrm{SiO}_{2}$, the analytical results match well; in fact, for some important elements (e.g., $\mathrm{FeO}^{*}, \mathrm{CaO}$, and $\mathrm{K}_{2} \mathrm{O}$ ) they match perfectly. 
Table 4. Electron microprobe analyses of mafic and intermediate glassy tephra recovered at Holes 907 A (Miocene sequence), 908A, and 913B.

\begin{tabular}{|c|c|c|c|c|c|c|c|c|c|c|}
\hline Sample: & \multicolumn{2}{|c|}{$151-907 \mathrm{~A}-11 \mathrm{H}-5$} & \multicolumn{2}{|c|}{$151-907 \mathrm{~A}-11 \mathrm{H}-5$} & \multicolumn{2}{|c|}{$151-907 \mathrm{~A}-11 \mathrm{H}-6$} & \multicolumn{2}{|c|}{$151-907 \mathrm{~A}-11 \mathrm{H}-6$} & \multicolumn{2}{|c|}{$151-907 \mathrm{~A}-12 \mathrm{H}$} \\
\hline Interval $(\mathrm{cm})$ : & \multicolumn{2}{|c|}{$105-106,106-107$} & \multicolumn{2}{|c|}{$105-106,106-107$} & \multicolumn{2}{|c|}{$56-57$} & \multicolumn{2}{|c|}{$130-132$} & \multicolumn{2}{|c|}{$50-52$} \\
\hline Label: & \multicolumn{2}{|c|}{$2 \mathrm{a}($ pop. 1$)$} & \multicolumn{2}{|c|}{$2 b$ (pop. II) } & \multicolumn{2}{|c|}{4} & \multicolumn{2}{|c|}{5} & \multicolumn{2}{|c|}{7} \\
\hline Type: & \multicolumn{2}{|c|}{ DA } & \multicolumn{2}{|c|}{ DA } & \multicolumn{2}{|c|}{ AL } & \multicolumn{2}{|c|}{ EL } & \multicolumn{2}{|c|}{$\mathrm{Al}$} \\
\hline Particle/points: & \multicolumn{2}{|c|}{$6 f / 14 p$} & \multicolumn{2}{|c|}{$6 f / 14 p$} & \multicolumn{2}{|c|}{$7 f / 14 p$} & \multicolumn{2}{|c|}{$8 \mathrm{f} / 20 \mathrm{p}$} & \multicolumn{2}{|c|}{$12 f / 35 p$} \\
\hline & Average $t$ & St. dev. & Average $t$ & St. dev. & Average t & St. dev. & Average t & St. dev. & Average t & St. dev. \\
\hline $\begin{array}{l}\mathrm{SiO}_{2} \\
\mathrm{TiO}_{2} \\
\mathrm{Al}_{2} \mathrm{O}_{3} \\
\mathrm{FeO}^{*} \\
\mathrm{MnO} \\
\mathrm{MgO} \\
\mathrm{CaO} \\
\mathrm{Na}_{2} \mathrm{O} \\
\mathrm{K}_{2} \mathrm{O} \\
\mathrm{P}_{2} \mathrm{O}_{5}\end{array}$ & $\begin{array}{r}50.26 \\
2.78 \\
13.80 \\
12.98 \\
0.23 \\
5.80 \\
10.03 \\
2.80 \\
0.68 \\
0.12\end{array}$ & $\begin{array}{l}1.26 \\
0.64 \\
0.74 \\
2.14 \\
0.03 \\
0.78 \\
1.04 \\
0.28 \\
0.36 \\
0.06\end{array}$ & $\begin{array}{r}58.27 \\
2.09 \\
13.87 \\
10.36 \\
0.18 \\
3.32 \\
6.84 \\
3.37 \\
1.29 \\
0.07\end{array}$ & $\begin{array}{l}2.74 \\
0.25 \\
0.10 \\
1.14 \\
0.03 \\
0.65 \\
0.91 \\
0.18 \\
0.26 \\
0.02\end{array}$ & $\begin{array}{r}60.11 \\
1.37 \\
14.53 \\
10.39 \\
0.29 \\
1.69 \\
5.21 \\
4.00 \\
1.33 \\
0.23\end{array}$ & $\begin{array}{l}2.16 \\
0.22 \\
0.18 \\
1.15 \\
0.05 \\
0.35 \\
0.63 \\
0.10 \\
0.18 \\
0.08\end{array}$ & $\begin{array}{r}54.85 \\
1.97 \\
13.79 \\
12.93 \\
0.28 \\
2.25 \\
6.60 \\
3.67 \\
1.53 \\
0.93\end{array}$ & $\begin{array}{l}2.01 \\
0.38 \\
0.38 \\
1.07 \\
0.04 \\
0.47 \\
0.65 \\
0.17 \\
0.13 \\
0.23\end{array}$ & $\begin{array}{r}52.04 \\
2.95 \\
12.98 \\
13.65 \\
0.28 \\
3.54 \\
8.16 \\
3.27 \\
0.86 \\
0.61\end{array}$ & $\begin{array}{l}2.81 \\
0.50 \\
0.33 \\
1.25 \\
0.03 \\
0.81 \\
1.23 \\
0.35 \\
0.24 \\
0.16\end{array}$ \\
\hline Sum. (vol.-free) & 99.48 & & 99.66 & & 99.15 & & 98.81 & & 98.33 & \\
\hline $\begin{array}{l}\mathrm{SO}_{2} \\
\mathrm{~F} \\
\mathrm{Cl}\end{array}$ & $\begin{array}{l}0.15 \\
0.14 \\
0.05\end{array}$ & $\begin{array}{l}0.07 \\
0.03 \\
0.04\end{array}$ & $\begin{array}{l}0.10 \\
0.14 \\
0.04\end{array}$ & $\begin{array}{l}0.02 \\
0.04 \\
0.02\end{array}$ & $\begin{array}{l}0.09 \\
0.20 \\
0.05\end{array}$ & $\begin{array}{l}0.01 \\
0.05 \\
0.02\end{array}$ & $\begin{array}{l}0.14 \\
0.21 \\
0.12\end{array}$ & $\begin{array}{l}0.04 \\
0.02 \\
0.02\end{array}$ & $\begin{array}{l}0.13 \\
0.19 \\
0.03\end{array}$ & $\begin{array}{l}0.04 \\
0.04 \\
0.01\end{array}$ \\
\hline Total & 99.82 & & 99.95 & & 99.49 & & 99.28 & & 98.68 & \\
\hline Sample: & $151-907$ & $\mathrm{~A}-13 \mathrm{H}-1$ & $151-907 \mathrm{~A}$ & A-14H-2 & $151-907$ & $-15 \mathrm{H}-5$ & $151-907 A$ & $-15 \mathrm{H}-5$ & $151-907$ & $-15 \mathrm{H}-6$ \\
\hline Interval $(\mathrm{cm})$ : & 69 & & $30-$ & & 94 & & 94-95, & $96-97$ & 96 & \\
\hline Label: & 1 & & 17 & & 29( & & 29 (bas & + top) & 3 & \\
\hline Type: & A & & (AI & & (A & & (Al & & EI & \\
\hline Particle/points: & \multicolumn{2}{|c|}{$11 f / 44 p$} & \multicolumn{2}{|c|}{$5 f / 8 p$} & \multicolumn{2}{|c|}{$3 f / 9 p$} & $13 \mathrm{f} /$ & $3 p$ & $14 \mathrm{fl}$ & \\
\hline $\begin{array}{l}\mathrm{SiO}_{2} \\
\mathrm{TiO}_{2} \\
\mathrm{Al}_{2} \mathrm{O}_{3} \\
\mathrm{FeO}^{*} \\
\mathrm{MnO} \\
\mathrm{MgO} \\
\mathrm{CaO} \\
\mathrm{Na}_{2} \mathrm{O} \\
\mathrm{K}_{2} \mathrm{O} \\
\mathrm{P}_{2} \mathrm{O}_{5}\end{array}$ & $\begin{array}{c}\text { Average t } \\
51.61 \\
3.21 \\
13.60 \\
13.34 \\
0.30 \\
4.25 \\
8.07 \\
3.21 \\
0.95 \\
0.48\end{array}$ & $\begin{array}{c}\text { St. dev. } \\
1.74 \\
0.39 \\
0.32 \\
1.02 \\
0.03 \\
0.48 \\
0.65 \\
0.18 \\
0.14 \\
0.16\end{array}$ & $\begin{array}{c}\text { Average h } \\
54.13 \\
2.49 \\
13.32 \\
13.07 \\
0.27 \\
3.23 \\
7.33 \\
3.20 \\
1.03 \\
0.82\end{array}$ & $\begin{array}{c}\text { St. dev. } \\
2.72 \\
0.82 \\
0.29 \\
0.63 \\
0.03 \\
1.21 \\
1.48 \\
0.33 \\
0.33 \\
0.30\end{array}$ & $\begin{array}{c}\text { Average t } \\
53.86 \\
2.12 \\
13.77 \\
12.38 \\
0.26 \\
4.16 \\
8.19 \\
3.23 \\
0.80 \\
0.35\end{array}$ & $\begin{array}{r}\text { St. dev. } \\
2.00 \\
0.18 \\
0.05 \\
0.86 \\
0.02 \\
0.51 \\
0.63 \\
0.07 \\
0.15 \\
0.02\end{array}$ & $\begin{array}{c}\text { Average } \\
51.97 \\
2.67 \\
13.68 \\
13.66 \\
0.23 \\
4.53 \\
8.97 \\
3.07 \\
0.55 \\
0.41\end{array}$ & $\begin{array}{c}\text { St. dev. } \\
0.90 \\
0.12 \\
0.19 \\
0.41 \\
0.03 \\
0.16 \\
0.24 \\
0.11 \\
0.04 \\
0.07\end{array}$ & $\begin{array}{c}\text { Average } \\
49.24 \\
3.12 \\
12.72 \\
14.44 \\
0.24 \\
5.34 \\
9.89 \\
2.68 \\
0.44 \\
0.32\end{array}$ & $\begin{array}{c}\text { St. dev. } \\
0.37 \\
0.52 \\
0.51 \\
0.84 \\
0.03 \\
0.62 \\
0.60 \\
0.21 \\
0.11 \\
0.14\end{array}$ \\
\hline Sum.(vol.-free) & 99.01 & & 98.88 & & 99.11 & & 99.74 & & 98.44 & \\
\hline $\begin{array}{l}\mathrm{SO}_{2} \\
\mathrm{~F} \\
\mathrm{Cl}\end{array}$ & $\begin{array}{l}0.19 \\
0.15 \\
0.04\end{array}$ & $\begin{array}{l}0.05 \\
0.03 \\
0.01\end{array}$ & $\begin{array}{l}0.16 \\
0.19 \\
0.05\end{array}$ & $\begin{array}{l}0.04 \\
0.03 \\
0.03\end{array}$ & $\begin{array}{l}0.05 \\
0.12 \\
0.02\end{array}$ & $\begin{array}{l}0.03 \\
0.00 \\
0.00\end{array}$ & $\begin{array}{l}0.05 \\
0.17 \\
0.04\end{array}$ & $\begin{array}{l}0.02 \\
0.03 \\
0.01\end{array}$ & $\begin{array}{l}0.10 \\
0.14 \\
0.03\end{array}$ & $\begin{array}{l}0.04 \\
0.03 \\
0.02\end{array}$ \\
\hline Total & 99.39 & & 99.28 & & 99.30 & & 100.00 & & 98.71 & \\
\hline Sample: & $151-907$ & $-16 \mathrm{H}-2$ & $151-907 \mathrm{~A}$ & $-16 \mathrm{H}-2$ & $151-907$ & $-16 \mathrm{H}-4$ & $151-907 \mathrm{~A}$ & $-16 \mathrm{H}-4$ & $151-907$ & $-18 \mathrm{H}-2$ \\
\hline Interval $(\mathrm{cm})$ : & 65 & & $65-$ & & 77 & & 95 & & 146 & 50 \\
\hline Label: & $33 \mathrm{a}(\mathrm{p}$ & op. 1) & $33 \mathrm{t}$ & & 3 & & 38 & & 40 & \\
\hline Type: & D. & & DA & & E & & $\mathrm{EI}$ & & (D) & \\
\hline Particle/points: & $6 \mathrm{f} / 1$ & & $3 \mathrm{f} / 6$ & & $13 \mathrm{f} /$ & & $8 \mathrm{f} / 1$ & & $8 \mathrm{f} / 1$ & \\
\hline & Average & St. dev. & Average h & St. dev. & Average & St. dev. & Average & St. dev. & Average & St. dev. \\
\hline $\begin{array}{l}\mathrm{SiO}_{2} \\
\mathrm{TiO}_{2} \\
\mathrm{Al}_{2} \mathrm{O}_{3} \\
\mathrm{FeO}^{*} \\
\mathrm{MnO} \\
\mathrm{MgO} \\
\mathrm{CaO} \\
\mathrm{Na}_{2} \mathrm{O} \\
\mathrm{K}_{2} \mathrm{O} \\
\mathrm{P}_{2} \mathrm{O}_{5}\end{array}$ & $\begin{array}{r}50.60 \\
3.08 \\
13.17 \\
14.20 \\
0.26 \\
4.66 \\
9.34 \\
2.97 \\
0.56 \\
0.42\end{array}$ & $\begin{array}{l}0.19 \\
0.09 \\
0.29 \\
0.25 \\
0.03 \\
0.06 \\
0.11 \\
0.11 \\
0.05 \\
0.02\end{array}$ & $\begin{array}{r}54.80 \\
2.49 \\
13.69 \\
12.53 \\
0.22 \\
3.52 \\
7.77 \\
3.30 \\
0.82 \\
0.50\end{array}$ & $\begin{array}{l}1.66 \\
0.25 \\
0.10 \\
0.73 \\
0.04 \\
0.50 \\
0.59 \\
0.22 \\
0.09 \\
0.03\end{array}$ & $\begin{array}{r}49.53 \\
2.63 \\
11.36 \\
17.19 \\
0.26 \\
4.32 \\
9.45 \\
2.53 \\
0.19 \\
0.42\end{array}$ & $\begin{array}{l}0.42 \\
0.22 \\
0.37 \\
0.52 \\
0.02 \\
0.41 \\
0.33 \\
0.06 \\
0.04 \\
0.07\end{array}$ & $\begin{array}{r}49.07 \\
2.75 \\
13.48 \\
13.09 \\
0.23 \\
5.69 \\
10.73 \\
2.74 \\
0.35 \\
0.35\end{array}$ & $\begin{array}{l}0.48 \\
0.24 \\
0.25 \\
0.52 \\
0.05 \\
0.50 \\
0.56 \\
0.14 \\
0.06 \\
0.04\end{array}$ & $\begin{array}{r}50.74 \\
1.70 \\
13.18 \\
13.36 \\
0.21 \\
5.60 \\
10.52 \\
2.64 \\
0.36 \\
0.25\end{array}$ & $\begin{array}{l}0.71 \\
0.27 \\
0.36 \\
0.60 \\
0.06 \\
0.81 \\
0.88 \\
0.33 \\
0.26 \\
0.06\end{array}$ \\
\hline Sum.(vol,-free) & 99.25 & & 99.63 & & 97.88 & & 98.47 & & 98.56 & \\
\hline $\begin{array}{l}\mathrm{SO}_{2} \\
\mathrm{~F} \\
\mathrm{Cl}\end{array}$ & $\begin{array}{l}0.04 \\
0.13 \\
0.02\end{array}$ & $\begin{array}{l}0.02 \\
0.02 \\
0.00\end{array}$ & $\begin{array}{l}0.04 \\
0.17 \\
0.01\end{array}$ & $\begin{array}{l}0.05 \\
0.03 \\
0.01\end{array}$ & $\begin{array}{l}0.29 \\
0.17 \\
0.36\end{array}$ & $\begin{array}{l}0.06 \\
0.02 \\
0.10\end{array}$ & $\begin{array}{l}0.08 \\
0.11 \\
0.02\end{array}$ & $\begin{array}{l}0.03 \\
0.05 \\
0.01\end{array}$ & $\begin{array}{l}0.16 \\
0.11 \\
0.09\end{array}$ & $\begin{array}{l}0.08 \\
0.03 \\
0.04\end{array}$ \\
\hline Total & 99.45 & & 99.85 & & 98.70 & & 98.69 & & 98.91 & \\
\hline
\end{tabular}


Table 4 (continued).

\begin{tabular}{|c|c|c|c|c|c|c|c|c|c|c|}
\hline Sample & \multicolumn{2}{|c|}{$151-907 \mathrm{~A}-21 \mathrm{H}-3$} & \multicolumn{2}{|c|}{$151-907 \mathrm{~A}-21 \mathrm{H}-3$} & \multicolumn{2}{|c|}{$151-907 \mathrm{~A}-21 \mathrm{H}-5$} & \multicolumn{2}{|c|}{$151-907 \mathrm{~A}-21 \mathrm{H}-5$} & \multicolumn{2}{|c|}{$151-907 \mathrm{~A}-21 \mathrm{H}-6$} \\
\hline Interval (cm) & \multicolumn{2}{|c|}{$72-73$} & \multicolumn{2}{|c|}{$94-95$} & \multicolumn{2}{|c|}{$35-37$} & \multicolumn{2}{|c|}{$104-106$} & \multicolumn{2}{|c|}{$20-22$} \\
\hline Label & \multicolumn{2}{|c|}{46} & \multicolumn{2}{|c|}{47} & \multicolumn{2}{|c|}{48} & \multicolumn{2}{|c|}{49} & \multicolumn{2}{|c|}{50} \\
\hline Type & \multicolumn{2}{|c|}{$\mathrm{DA}$} & \multicolumn{2}{|c|}{ EL } & \multicolumn{2}{|c|}{ DA } & \multicolumn{2}{|c|}{ DA } & \multicolumn{2}{|c|}{$\mathrm{DA}$} \\
\hline Particle/points & \multicolumn{2}{|c|}{$5 \mathrm{f} / 11 \mathrm{p}$} & \multicolumn{2}{|c|}{$7 f / 13 p$} & \multicolumn{2}{|c|}{$5 f / 15 p$} & \multicolumn{2}{|c|}{$10 / 53 p$} & \multicolumn{2}{|c|}{$4 f / 12 p$} \\
\hline & Average & St. dev. & Average & St. dev, & Average & St. dev, & Average & St. dev. & Average & St. dev. \\
\hline $\begin{array}{l}\mathrm{SiO}_{2} \\
\mathrm{TiO}_{2} \\
\mathrm{Al}_{2} \mathrm{O}_{3} \\
\mathrm{FeO}^{*} \\
\mathrm{MnO} \\
\mathrm{MgO} \\
\mathrm{CaO} \\
\mathrm{Na}_{2} \mathrm{O} \\
\mathrm{K}_{2} \mathrm{O} \\
\mathrm{P}_{2} \mathrm{O}_{5}\end{array}$ & $\begin{array}{r}50.53 \\
1.80 \\
13.06 \\
14.37 \\
0.20 \\
6.42 \\
10.64 \\
2.39 \\
0.09 \\
0.20\end{array}$ & $\begin{array}{l}0.53 \\
0.14 \\
0.19 \\
0.68 \\
0.03 \\
0.48 \\
0.53 \\
0.08 \\
0.03 \\
0.02\end{array}$ & $\begin{array}{r}49.39 \\
2.06 \\
13.01 \\
13.89 \\
0.27 \\
6.04 \\
10.80 \\
2.41 \\
0.11 \\
0.24\end{array}$ & $\begin{array}{l}0.31 \\
0.57 \\
0.46 \\
1.29 \\
0.04 \\
0.63 \\
0.76 \\
0.21 \\
0.12 \\
0.05\end{array}$ & $\begin{array}{r}49.59 \\
2.10 \\
12.79 \\
15.21 \\
0.27 \\
5.84 \\
10.26 \\
2.36 \\
0.13 \\
0.17\end{array}$ & $\begin{array}{l}0.53 \\
0.55 \\
0.52 \\
1.48 \\
0.04 \\
0.67 \\
0.53 \\
0.09 \\
0.05 \\
0.04\end{array}$ & $\begin{array}{r}49.45 \\
1.73 \\
13.68 \\
13.23 \\
0.20 \\
6.99 \\
11.59 \\
2.36 \\
0.12 \\
0.15\end{array}$ & $\begin{array}{l}0.52 \\
0.37 \\
0.50 \\
1.19 \\
0.02 \\
0.48 \\
0.49 \\
0.15 \\
0.10 \\
0.04\end{array}$ & $\begin{array}{r}50.26 \\
1.32 \\
13.64 \\
13.09 \\
0.29 \\
6.81 \\
11.23 \\
2.39 \\
0.17 \\
0.20\end{array}$ & $\begin{array}{l}0.24 \\
0.34 \\
0.57 \\
0.72 \\
0.05 \\
0.74 \\
0.51 \\
0.36 \\
0.19 \\
0.06\end{array}$ \\
\hline Sum. (vol.-free) & 99.70 & & 98.21 & & 98.72 & & 99.50 & & 99.39 & \\
\hline $\begin{array}{l}\mathrm{SO}_{2} \\
\mathrm{~F} \\
\mathrm{Cl}\end{array}$ & $\begin{array}{l}0.22 \\
0.10 \\
0.15\end{array}$ & $\begin{array}{l}0.03 \\
0.04 \\
0.05\end{array}$ & $\begin{array}{l}0.26 \\
0.13 \\
0.14\end{array}$ & $\begin{array}{l}0.08 \\
0.03 \\
0.05\end{array}$ & $\begin{array}{l}0.19 \\
0.10 \\
0.10\end{array}$ & $\begin{array}{l}0.04 \\
0.02 \\
0.06\end{array}$ & $\begin{array}{l}0.18 \\
0.09 \\
0.07\end{array}$ & $\begin{array}{l}0.05 \\
0.02 \\
0.05\end{array}$ & $\begin{array}{l}0.18 \\
0.09 \\
0.03\end{array}$ & $\begin{array}{l}0.08 \\
0.05 \\
0.02\end{array}$ \\
\hline Total & 100.17 & & 98.74 & & 99.10 & & 99.84 & & 99.69 & \\
\hline Sample & \multicolumn{2}{|c|}{$151-907 \mathrm{~A}-21 \mathrm{H}-6$} & \multicolumn{2}{|c|}{$151-908 \mathrm{~A}-17 \mathrm{X}-4$} & & & & & & \\
\hline Interval $(\mathrm{cm})$ & \multicolumn{2}{|c|}{$51-52$} & \multicolumn{2}{|c|}{$6-7,7-8$} & & & & & & \\
\hline Label & \multicolumn{2}{|c|}{51} & \multicolumn{2}{|c|}{52} & & & & & & \\
\hline Type & \multicolumn{2}{|c|}{ DA } & \multicolumn{2}{|c|}{ (AL) } & & & & & & \\
\hline \multirow[t]{2}{*}{ Particle/points } & $8 \mathrm{f} / 1$ & & $14 \mathrm{f} / 2$ & $0 \mathrm{p}$ & & & & & & \\
\hline & Average & St. dev. & Average t & St. dev. & & & & & & \\
\hline $\begin{array}{l}\mathrm{SiO}_{2} \\
\mathrm{TiO}_{2} \\
\mathrm{Al}_{2} \mathrm{O}_{3} \\
\mathrm{FeO}^{*} \\
\mathrm{MnO} \\
\mathrm{MgO} \\
\mathrm{CaO} \\
\mathrm{Na}_{2} \mathrm{O} \\
\mathrm{K}_{2} \mathrm{O} \\
\mathrm{P}_{2} \mathrm{O}_{5}\end{array}$ & $\begin{array}{r}49.86 \\
1.79 \\
13.28 \\
13.32 \\
0.24 \\
6.22 \\
11.10 \\
2.47 \\
0.13 \\
0.21\end{array}$ & $\begin{array}{l}0.61 \\
0.33 \\
0.31 \\
0.63 \\
0.02 \\
0.17 \\
0.33 \\
0.19 \\
0.11 \\
0.05\end{array}$ & $\begin{array}{r}57.77 \\
1.54 \\
13.27 \\
12.09 \\
0.31 \\
1.75 \\
5.98 \\
3.57 \\
1.22 \\
0.80\end{array}$ & $\begin{array}{l}2.36 \\
0.37 \\
0.24 \\
1.58 \\
0.05 \\
0.62 \\
0.84 \\
0.26 \\
0.17 \\
0.36\end{array}$ & & & & & & \\
\hline Sum.(vol.-free) & 98.63 & & 98.29 & & & & & & & \\
\hline $\begin{array}{l}\mathrm{SO}_{2} \\
\mathrm{~F} \\
\mathrm{Cl}\end{array}$ & $\begin{array}{l}0.20 \\
0.12 \\
0.08\end{array}$ & $\begin{array}{l}0.06 \\
0.03 \\
0.08\end{array}$ & $\begin{array}{l}0.15 \\
0.21 \\
0.06\end{array}$ & $\begin{array}{l}0.13 \\
0.05 \\
0.02\end{array}$ & & & & & & \\
\hline Total & 99.02 & & 98.70 & & & & & & & \\
\hline
\end{tabular}

Note: $\mathrm{AL}=$ discrete ash layer, $\mathrm{DA}=$ disseminated ash layer, $\mathrm{EL}=$ ash-enriched layer; type in parentheses $(\mathrm{AL})=$ subordinate mafic or intermediate population in a silicic layer; particles/points $=$ number of analyzed particles $(\mathrm{f})$ and total number of analyses $(\mathrm{p})$; average $\mathrm{t}=$ average of a group of analyses showing a clear trend; average $\mathrm{h}=\mathrm{average}$ of a group of chemical heterogeneous particles in an individual deposit.

\section{Rhyolitic and Trachytic Tephra}

Most of the Miocene rhyolitic glass shards (Holes 907A and 908A) are compositionally homogeneous (Figs. 3, 4). Only some glass particles found in the middle Miocene sequence in Hole 907A (open squares in Fig. 4) have somewhat higher $\mathrm{K}_{2} \mathrm{O}$ and lower $\mathrm{CaO}$ and $\mathrm{FeO}^{*}$ concentrations than the majority of the Miocene tephra. By contrast, the late Eocene to early Oligocene tephra (Hole 913B, open diamonds in Fig. 4) have a distinct higher $\mathrm{Al}_{2} \mathrm{O}_{3}$ and $\mathrm{K}_{2} \mathrm{O}$ and lower $\mathrm{SiO}_{2}$ content compared to the Miocene tephra.

Sigurdsson and Loebner (1981) distinguish two series of Cenozoic tephra in the North Atlantic region: a high potassium series $\left(\mathrm{K}_{2} \mathrm{O}\right.$ $>3.8 \mathrm{wt} \%$ ) that ranges from quartz trachytes to alkali rhyolites and a low potassium series $\left(\mathrm{K}_{2} \mathrm{O}>3.8 \mathrm{wt} \%\right)$ that ranges from icelandites to rhyolites. The majority of the rhyolitic glass shards analyzed belong to the low potassium series. They are rhyolites containing 11.5-14 wt $\% \mathrm{Al}_{2} \mathrm{O}_{3}, 0.8-2.5 \mathrm{wt} \% \mathrm{CaO}$, and $2-5$ wt $\% \mathrm{FeO}^{*}$. The remaining shards belong to the high potassium series and are alkali rhyolites with $12-15$ wt $\% \mathrm{Al}_{2} \mathrm{O}_{3},<1 \mathrm{wt} \% \mathrm{CaO}$, and $1-4 \mathrm{wt} \% \mathrm{FeO} *$ (all values calculated on a water-free basis). Alkali rhyolitic shards occur almost exclusively in the oldest ash deposits of Hole 907A (layers 45 and 48 in Cores $151-907 \mathrm{~A}-20 \mathrm{H}$ and $21 \mathrm{H}$ ) as well as in the ash layer in Hole 913B. Peralkaline rhyolites as described by Lacasse et al. (this volume) for the Pliocene and Pleistocene sequence of Hole 907A do not occur in the Miocene deposits.

The trachytic shards of layer 9 (Core $151-907 \mathrm{~A}-12 \mathrm{H})$ are characterized by high concentrations of $\mathrm{Al}_{2} \mathrm{O}_{3}, \mathrm{~K}_{2} \mathrm{O}, \mathrm{FeO} *$ (Fig. 4), and $\mathrm{Na}_{2} \mathrm{O}(6.5-7.0 \mathrm{wt} \%$ on a water-free base). This trachytic ash layer clearly shows two chemically different populations, which differ particularly in $\mathrm{Si}, \mathrm{Fe}$, and $\mathrm{Ca}$ content.

\section{Basaltic to Dacitic Tephra}

Figure 5 shows analyses of individual mafic to intermediate glass shards with $\mathrm{SiO}_{2}, \mathrm{Al}_{2} \mathrm{O}_{3}, \mathrm{CaO}, \mathrm{FeO} *, \mathrm{~K}_{2} \mathrm{O}$, and $\mathrm{TiO}_{2}$ vs. decreasing $\mathrm{MgO}$. Almost all analyzed particles belong to the sub-alkalic and low-K sub-alkalic series, respectively. The basaltic to dacitic glass particles recovered in Holes 907A and 908A differ in chemical composition and are characterized by large compositional ranges.

The late Miocene tephra recovered in Holes 907A and 908A always plot in the same field but cover a broad spectrum from sub-alkalic basaltic to dacitic compositions ranging from 0.6 to $6.6 \mathrm{wt} \%$ in 


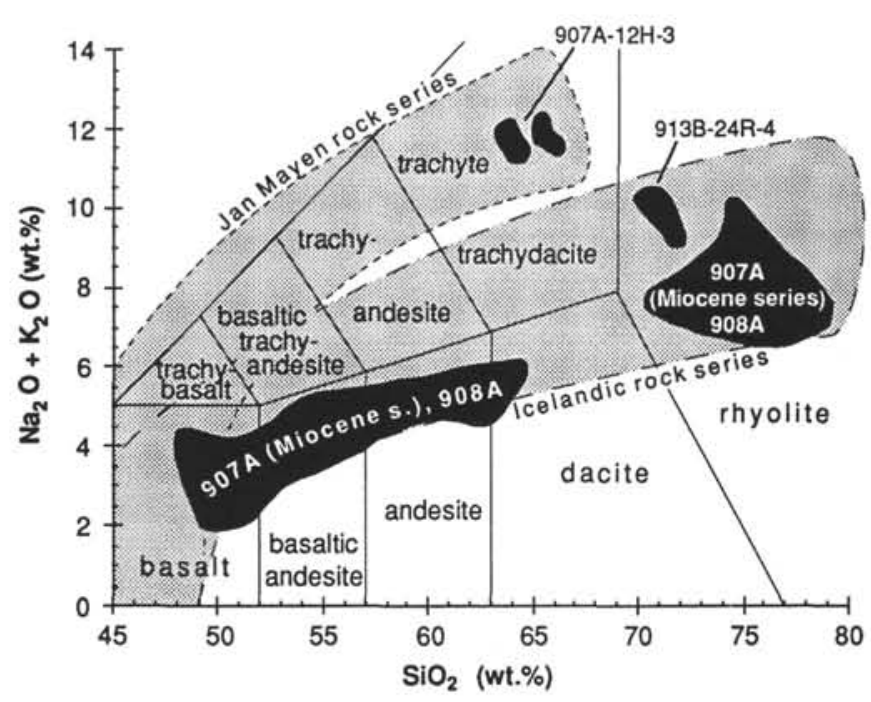

Figure 3. Classification of the glass component of tephra recovered in Holes 907A (Miocene sequence), 908A, and 913B (black fields) based on total alkali-silica plot (after Le Bas et al., 1986). Fields for Jan Mayen rock series after Imsland (1984), for Icelandic rock series after Jakobsson (1979). All analyses have been normalized to a total of $100 \%$ (volatile-free basis, with $\mathrm{Fe}$ calculated as $\mathrm{FeO}^{*}$ ).
$\mathrm{MgO}, 1$ to $5 \mathrm{wt} \%$ in $\mathrm{TiO}_{2}$, and 12.5 to $15.2 \mathrm{wt} \%$ in $\mathrm{Al}_{2} \mathrm{O}_{3}$ concentrations. All analyses of the late Miocene glass particles show a positive correlation for $\mathrm{SiO}_{2}$ and $\mathrm{K}_{2} \mathrm{O}$ vs. $\mathrm{MgO}$ and a negative correlation for $\mathrm{CaO}, \mathrm{FeO} *$, and $\mathrm{TiO}_{2}$ vs. $\mathrm{MgO}$.

Most of the mafic to intermediate glass particles found on top of the middle Miocene sequence (Cores 151-907A-15H and $16 \mathrm{H}$ ) are basaltic, and nearly half of them belong to the low-K sub-alkali series. Their $\mathrm{MgO}$ concentrations range from 3 to $7 \mathrm{wt} \%, \mathrm{TiO}_{2}$ from 1.5 to $4 \mathrm{wt} \%$, and $\mathrm{Al}_{2} \mathrm{O}_{3}$ from 11.2 to $14 \mathrm{wt} \%$. All analyses show a negative correlation for $\mathrm{CaO}$ and partly a negative correlation for $\mathrm{Al}_{2} \mathrm{O}_{3}$ vs. $\mathrm{MgO}$. Two groups can be distinguished in the $\mathrm{K}_{2} \mathrm{O}$ and $\mathrm{FeO}^{*}$ vs. $\mathrm{MgO}$ plots: a group with the higher $\mathrm{K}_{2} \mathrm{O}$ contents $(0.3-1 \mathrm{wt} \%$ ) has medium $\mathrm{FeO}^{*}$ contents $(10.6-15.8 \mathrm{wt} \%)$, and a group with relative low $\mathrm{K}_{2} \mathrm{O}$ contents $(0.06-0.3)$ is characterized by extremely high $\mathrm{FeO}^{*}$ concentrations $(>16 \mathrm{wt} \%)$. All shards belonging to the second group were found exclusively in ash-enriched layer 37 (Core 151907A-16H).

The rest of the middle Miocene mafic ash deposits (Cores 151$907 \mathrm{~A}-18 \mathrm{H}$ to $21 \mathrm{H}$ ) consist only of basaltic particles with low-K subalkalic affinity except for those belonging to layer 40 (Core 151$907 \mathrm{~A}-18 \mathrm{H})$. They range between 5.5 and $8.5 \mathrm{wt} \%$ in $\mathrm{MgO}$ and follow roughly the path for plagioclase and olivine fractionation for $\mathrm{TiO}_{2}$ vs. $\mathrm{MgO}$. Similar to the results of analyses of the series on top of the middle Miocene sequence, these analyses show a clear correlation for $\mathrm{CaO}$ vs. $\mathrm{MgO}$ and two different trends in the $\mathrm{K}_{2} \mathrm{O}$ vs. $\mathrm{MgO}$ plot, but they have relatively higher $\mathrm{CaO}$ and lower $\mathrm{K}_{2} \mathrm{O}$ concentrations. The

Table 5. Comparison of major element analyses of the same silicic and mafic glassy tephra.

\begin{tabular}{|c|c|c|c|c|c|c|c|c|}
\hline \multirow{3}{*}{$\begin{array}{c}\text { Sample: } \\
\text { Interval }(\mathrm{cm}):\end{array}$} & \multicolumn{4}{|c|}{$151-907 \mathrm{~A}-10 \mathrm{H}-1$} & \multicolumn{4}{|c|}{$151-907 \mathrm{~A}-10 \mathrm{H}-2$} \\
\hline & \multicolumn{2}{|c|}{$148-149$} & \multicolumn{2}{|c|}{$148-150$} & \multicolumn{2}{|c|}{$4-5,8-9$} & \multicolumn{2}{|c|}{$0-2$} \\
\hline & \multicolumn{2}{|c|}{ this study } & \multicolumn{2}{|c|}{$\begin{array}{l}\text { Lacasse et al. } \\
\text { (this volume) }\end{array}$} & \multicolumn{2}{|c|}{ this study } & \multicolumn{2}{|c|}{$\begin{array}{l}\text { Lacasse et al. } \\
\text { (this volume) }\end{array}$} \\
\hline \multirow[t]{2}{*}{ Particles/points: } & \multicolumn{2}{|c|}{$4 f / 10 p$} & \multicolumn{2}{|c|}{$14 p$} & \multicolumn{2}{|c|}{$13 \mathrm{f} / 40 \mathrm{p}$} & \multicolumn{2}{|c|}{$15 p$} \\
\hline & Average & St. dev. & Average & St. dev. & Average & St. dev. & Average & St. dev. \\
\hline $\begin{array}{l}\mathrm{SiO}_{2} \\
\mathrm{TiO}_{2} \\
\mathrm{Al}_{2} \mathrm{O}_{3} \\
\mathrm{FeO}^{*} \\
\mathrm{MnO} \\
\mathrm{MgO} \\
\mathrm{CaO} \\
\mathrm{Na}_{2} \mathrm{O} \\
\mathrm{K}_{2} \mathrm{O}\end{array}$ & $\begin{array}{r}71.91 \\
0.15 \\
12.45 \\
2.31 \\
0.08 \\
0.00 \\
1.08 \\
5.07 \\
2.37\end{array}$ & $\begin{array}{l}0.61 \\
0.02 \\
0.08 \\
0.02 \\
0.04 \\
0.00 \\
0.03 \\
0.12 \\
0.12\end{array}$ & $\begin{array}{r}71.08 \\
0.17 \\
12.24 \\
2.28 \\
0.08 \\
0.01 \\
1.14 \\
5.44 \\
2.41\end{array}$ & $\begin{array}{l}0.49 \\
0.04 \\
0.19 \\
0.15 \\
0.05 \\
0.01 \\
0.07 \\
0.29 \\
0.13\end{array}$ & $\begin{array}{r}70.52 \\
0.13 \\
12.08 \\
2.20 \\
0.10 \\
0.01 \\
1.10 \\
5.11 \\
2.36\end{array}$ & $\begin{array}{l}0.22 \\
0.05 \\
0.09 \\
0.06 \\
0.03 \\
0.01 \\
0.02 \\
0.10 \\
0.06\end{array}$ & $\begin{array}{r}71.49 \\
0.14 \\
12.18 \\
2.23 \\
0.09 \\
0.01 \\
1.11 \\
5.36 \\
2.38\end{array}$ & $\begin{array}{l}0.43 \\
0.04 \\
0.12 \\
0.14 \\
0.05 \\
0.01 \\
0.04 \\
0.16 \\
0.09\end{array}$ \\
\hline Total & 95.43 & & 94.85 & & 93.60 & & 94.99 & \\
\hline
\end{tabular}

\begin{tabular}{|c|c|c|c|c|}
\hline \multirow{4}{*}{$\begin{array}{c}\text { Sample: } \\
\text { Interval }(\mathrm{cm}) \\
\text { Particles/points: }\end{array}$} & \multicolumn{4}{|c|}{$151-907 \mathrm{~A}-10 \mathrm{H}-1$} \\
\hline & \multicolumn{2}{|c|}{$148-149$} & \multicolumn{2}{|c|}{$148-150$} \\
\hline & \multicolumn{2}{|c|}{ this study } & \multicolumn{2}{|c|}{$\begin{array}{l}\text { Lacasse et al. } \\
\text { (this volume) }\end{array}$} \\
\hline & \multicolumn{2}{|c|}{$5 f / 10 p$} & \multicolumn{2}{|c|}{$15 p$} \\
\hline & Average & St. dev. & Average & St. dev. \\
\hline $\mathrm{SiO}_{2}$ & 48.39 & 0.14 & 48.88 & 0.37 \\
\hline $\mathrm{TiO}_{2}$ & 3.12 & 0.11 & 3.14 & 0.12 \\
\hline $\mathrm{Al}_{2} \mathrm{O}_{3}$ & 12.91 & 0.08 & 13.26 & 0.16 \\
\hline $\mathrm{FeO}^{*}$ & 15.16 & 0.11 & 15.20 & 0.22 \\
\hline $\mathrm{MnO}$ & 0.29 & 0.02 & 0.23 & 0.05 \\
\hline $\mathrm{MgO}$ & 5.39 & 0.07 & 5.45 & 0.12 \\
\hline $\mathrm{CaO}$ & 10.32 & 0.07 & 10.30 & 0.16 \\
\hline $\mathrm{Na}_{2} \mathrm{O}$ & 2.80 & 0.04 & 2.71 & 0.09 \\
\hline $\mathrm{K}_{2} \mathrm{O}$ & 0.44 & 0.03 & 0.44 & 0.03 \\
\hline $\mathrm{P}_{2} \mathrm{O}_{5}$ & 0.40 & 0.02 & 0.46 & 0.04 \\
\hline Total & 99.22 & & 100.07 & \\
\hline
\end{tabular}

Notes: Analyses carried out with Cameca SX 50 electron microprobe at GEOMAR, Kiel, Federal Republic of Germany (this study) and Cameca electron microprobe at Brown University, Rhode Island, U.S.A. (Lacasse et al,, this volume); particles/points = number of analyzed particles (f) and total number of analyses (p). 

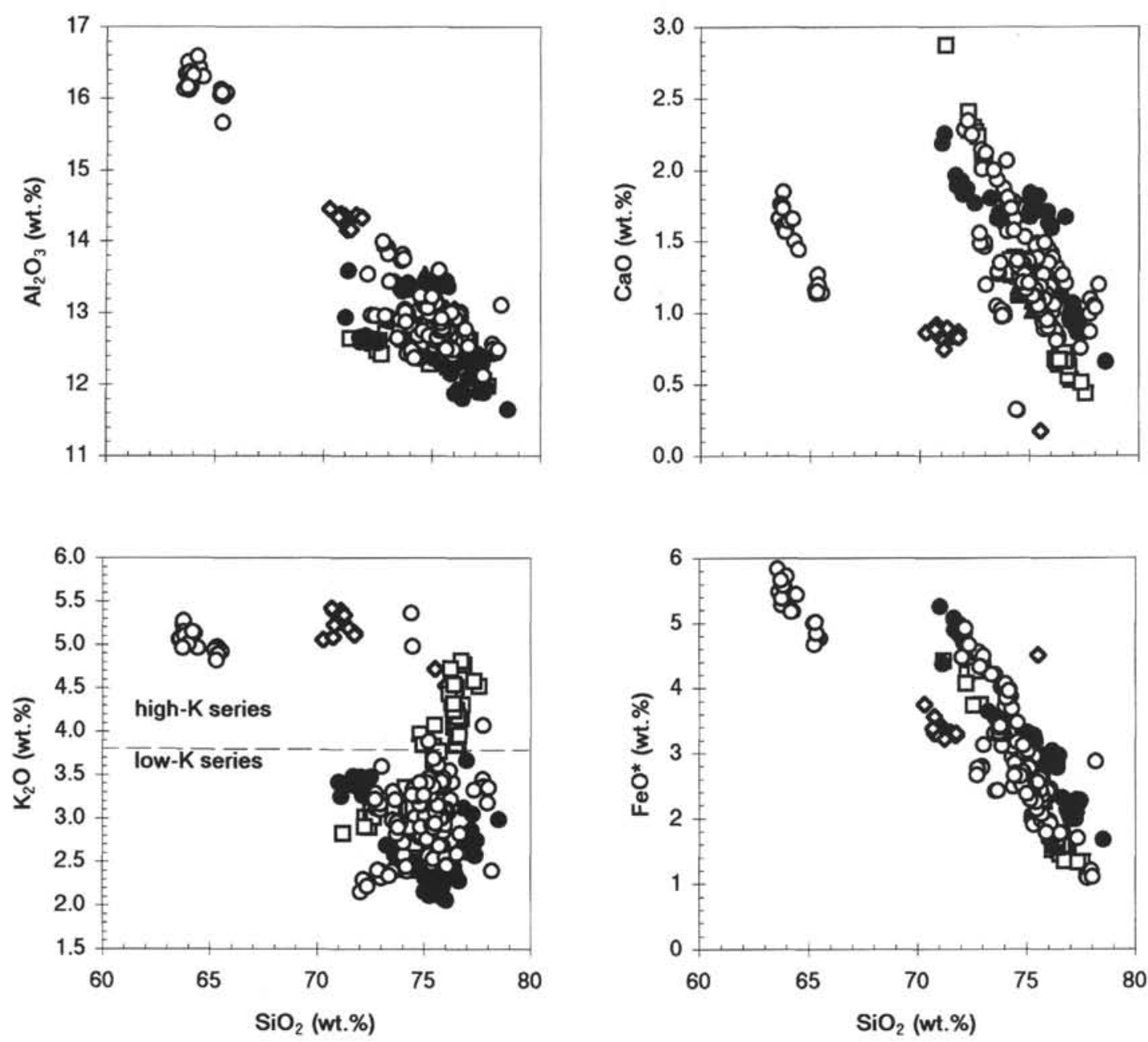

Figure 4. Major element composition $\left(\mathrm{Al}_{2} \mathrm{O}_{3}, \mathrm{CaO}, \mathrm{FeO} * \mathrm{~K}_{2} \mathrm{O}\right)$ and subdivision in low- $\mathrm{K}$ and high-K series (after Sigurdsson and Loebner, 1981) of rhyolitic and trachytic glass particles recovered in Cores 151-907A-11H to $14 \mathrm{H}$ (late Miocene; open circles), Cores $151-907 \mathrm{~A}-15 \mathrm{H}$ and $16 \mathrm{H}$ (on top of the middle Miocene sequence; solid circles), Cores 151-907A-18H to 21H (middle Miocene; open squares), Core 151-908A-17X (late Miocene to early Pliocene (?), solid triangles) and in Core 151-913B-24R (late Eocene to early Oligocene, open diamonds). Plots are vs. increasing $\mathrm{SiO}_{2}$ concentration. All analyses have been normalized to a total of $100 \%$ (volatile-free basis, with $\mathrm{Fe}$ calculated as $\mathrm{FeO}^{*}$ )

variation of $\mathrm{FeO}^{*}$ vs. $\mathrm{MgO}$ fits with the early stages of fractionation with increasing iron enrichment in the Thingmuli trend (Carmichael, 1964).

\section{SULFUR CONTENT AND DEGREE OF DEGASSING}

The sulfur content of the analyzed mafic and intermediate glass particles ranges from 50 to $2000 \mathrm{ppm}$ (Fig. 6A). The tephra recovered in Hole $908 \mathrm{~A}$ and on top of the middle Miocene sequence of Hole 907A (Cores 151-907 A-15H and 16H) show the lowest sulfur contents with an average of 533 and $578 \mathrm{ppm}$, respectively. The highest contents, with an average of $966 \mathrm{ppm}$, are found for the oldest ashbearing layers of Hole 907A (Cores $151-907 \mathrm{~A}-18 \mathrm{H}$ to $21 \mathrm{H}$ ). The average sulfur content of the tephra on top of the middle Miocene sequence of Hole 907A (Cores 151-907A-15H and 16H) amounts to $736 \mathrm{ppm}$.

The most important factor controlling degassing and, therefore, the sulfur concentration of the mafic tephra is the position of the eruption center relative to the water level because high hydrostatic pressure prevents the escape of volatile elements by exsolution from erupted lava. According to Moore and Schilling (1973), the sulfur concentration of submarine lavas decreases rapidly when the eruptive center is situated in a water depth of less than $200 \mathrm{~m}$; whereas, below $200 \mathrm{~m}$, sulfur concentration is independent from depth. Similar values are reported from Moore and Calk (1991) and Werner (1994) for sublacustrine and subglacial erupted lavas. Calculations by Gerlach (1986) indicate that approximately $20 \mathrm{wt} \%$ of the sulfur will degas at $300 \mathrm{~m}$ depth and $40 \mathrm{wt} \%$ at $100 \mathrm{~m}$ depth. Another factor that may inhibit sulfur degassing is quenching. For example, the sulfur concentration of glassy ash erupted during a small subglacial eruption in Iceland is somewhat higher ( $760 \mathrm{ppm})$ than that of other phreatomagmatic eruptions, owing probably to rapid quenching (Grönvold and Johannesson, 1984; Moore and Calk, 1991). In contrast to products of deep submarine eruptions, which have uniformly high (original) sulfur values, products of shallow water and subglacial eruptions usually show a broad range of sulfur concentration because of changing eruption environments (e.g., Werner, 1994). Metrich et al. (1991) point out that sulfur concentrations of matrix glasses decrease significantly with progressive differentiation or increasing $\mathrm{FeO}^{*}$ concentration. Using $\mathrm{MgO}$ as abscissa as an index of differentiation, a correlation between the degree of differentiation and sulfur concentrations is not apparent for the mafic and intermediate glass particles recovered at Holes 907A and 908A (Fig. 6A). On the other hand, a 



Figure 5. Major element composition $\left(\mathrm{SiO}_{2}, \mathrm{Al}_{2} \mathrm{O}_{3}, \mathrm{CaO}, \mathrm{FeO} *, \mathrm{~K}_{2} \mathrm{O}, \mathrm{TiO}_{2}\right.$ ) of basaltic and dacitic glass particles recovered in Cores $151-907 \mathrm{~A}-11 \mathrm{H}$ to $14 \mathrm{H}$ (late Miocene; open circles), Cores 151-907A-15H and 16H (on top of the middle Miocene sequence; solid circles), Cores 151-907A-18H to 21H (middle Miocene; open squares) and in Core 151-908A-17X (late Miocene to early Pliocene (?), solid triangles). Plots are vs. decreasing MgO concentration. All analyses have been normalized to a total of $100 \%$ (volatile-free basis, with Fe calculated as FeO*). The Thingmuli tholeiitic trend (Carmichael, 1964) is represented by the arrows. 
correlation between $\mathrm{FeO}^{*}$ and sulfur clearly exists (Fig. 6B). This correlation appears mainly caused, however, by tephra with extremely high $(>16 \mathrm{wt} \%)$ and low $(<12.5 \mathrm{wt} \%) \mathrm{FeO}^{*}$ concentrations. Considering only the glass particles with 12.5 to $16 \mathrm{wt} \% \mathrm{FeO}^{*}$, which make up the majority of analyzed particles, $\mathrm{FeO}^{*}$ and $\mathrm{S}$ concentrations do not correlate (Fig. 6C). Furthermore a negative correlation of sulfur concentrations of matrix glasses and vesicularity is shown by Metrich et al. (1991). But loss of the major volatile phases and, therefore, vesiculation also strongly depends on the hydrostatic pressure (e.g., Fisher and Schmincke, 1984). For instance, actual loss of water, the most abundant volatile phase in tholeiitic basaltic magmas, by vesiculation in ocean-floor tholeiites takes place only above about 200 $\mathrm{m}$ water depths (e.g., Moore and Schilling, 1973).

Consequently, most sulfur analyses presented in this study can be used to evaluate the relative degree of degassing of tephra and from that the paleoenvironment at the time of eruption. According to several authors (e.g., Moore and Fabbi, 1971; Moore and Schilling, 1973; Moore and Calk, 1991; Metrich et al., 1991; Werner, 1994) the original sulfur concentrations of deep-sea basalts as well as of Icelandic tholeiitic basaltic magmas clearly exceed $800 \mathrm{ppm}$. Therefore, all analyzed glass particles with medium $\mathrm{FeO}^{*}$ values and sulfur concentrations $<600$ ppm (below unbroken line in Fig. 6A) are significantly degassed with regard to sulfur, whereas sulfur concentrations $>900$ ppm (above dashed line in Fig. 6A) indicate relatively minor degassing and may partly represent the original sulfur concentrations of the magmas.

\section{DISCUSSION \\ Eruption Processes, Environment, and Mode of Transport}

The rhyolitic and trachytic ash deposits contain mainly bubble wall shards; pumice fragments are also common in most deposits. These shards are clearly formed by vesiculation (pyroclastic fragmentation). For this reason, and because of the good sorting, the relatively small grain size, and the lack of (dense) crystals, these deposits are interpreted as distal fallout deposits from high eruption columns of Plinian eruptions (e.g., Fisher and Schmincke, 1984). Normal and inverse grading observed in layers 19,23, and 29 (Table 1) can be attributed to fluctuating eruption columns or size fragmentation in the water column of more or less uniform shards and, for example, pumice fragments. Bimodal compositions of some deposits are probably caused by eruptions from zoned magma columns as are the trends from rhyolitic, dacitic or andesitic to more mafic endmembers in layers $2,4,5,7,11,17(?), 29$, and possibly 52 (Table 3).

The light brown glass particles in ash deposits with intermediate composition (e.g., layers 5,7 , or 11 ) are also mostly vesicular or pumice-like (Table 1) and therefore probably produced by pyroclastic processes. At least layer 7, however, also contains a significant amount of dense blocky shards typical of hydroclastic fragmentation processes (steam explosions, cooling granulation). The coexistence of partly highly vesicular and dense blocky particles is also typical for several pure basaltic ash deposits. Considering the sulfur contents of mafic and intermediate glass particles (Figs. 6, 7A) recovered in Cores $151-907 \mathrm{~A}-11 \mathrm{H}$ to $18 \mathrm{H}$ (layers no. $2-40$ ), it is obvious that sulfur loss was relatively minor from a part of these particles. The different texture and the varying degree of degassing of glass particles in single ash deposits might be explained in two ways. First, these deposits are products of a submarine volcano growing from relatively deep water ( $>200 \mathrm{~m}$ water depth) to the surface. Second, they are products of subglacial/sublacustrine volcanoes. More or less undegassed blocky shards were produced by cooling granulation during the deep-water stage and the subglacial stage in a water-filled ice cave, respectively. In the shallow-water and emerged stage, explosive hydroclastic eruptions take place and might also initiate pyro- clastic eruptions (e.g., Fröhlich et al., 1990; Werner, 1994). From these processes result a broad spectrum of highly vesicular to dense particles with varying degrees of degassing.

More than $90 \%$ of the analyzed basaltic particles in Core 151907A-21H (layers 46-51) show high sulfur concentrations and, therefore, relatively low degrees of degassing (Fig 7B). Most of these particles are blocky and dense or have small vesicles. This indicates a submarine origin by cooling granulation in water depths of much greater than $200 \mathrm{~m}$. The vesicles might be formed by exsolution of, for example, $\mathrm{CO}_{2}$, which already takes place in water depths greater than $4800 \mathrm{~m}$ (Moore et al., 1977). All ash deposits recovered in this core are disseminated ash layers or ash-enriched layers containing around $10 \%-60 \%$ glass particles. They are predominantly poorly sorted with large maximum grain sizes (Table 1) and therefore were probably deposited by mass flows or turbidity currents.

\section{Stratigraphy}

Site 907 is located on oceanic crust, 22-24 Ma in age (magnetic anomaly 6B, Shipboard Scientific Party, 1995a), nearly halfway between the active Kolbeinsey Ridge and the Jan Mayen Ridge and halfway between Iceland and Jan Mayen Island (Fig. 1). The cores record volcanic input since middle Miocene. The oldest ash layers of Hole 907A (around 14 Ma old) were deposited at a time when the site was located near the newly established Kolbeinsey Ridge compared with the plate reconstructions by Nunns (1983).

Site 913 , today located in the deep Greenland Basin, rests on crust older than $55 \mathrm{Ma}$ (anomaly 24B, Shipboard Scientific Party, 1995c), the oldest oceanic crust present in the Norwegian-Greenland Sea. The two ash layers found in Cores 151-913B-24R and 26R are late Eocene to early Oligocene in age. During this time, the Greenland Basin was much smaller than it is today, and the distance of the site to the ancient active spreading center was around half of the recent distance to the Mohns Ridge (around $500 \mathrm{~km}$ ). East of the site, some poorly known volcanic explosive activities (related to the rifting of the Jan Mayen Ridge from East Greenland during the Oligocene), which produced rhyolitic material, were estimated by Sigurdsson and Loebner (1981). The ashes of Hole 913B may correlate with these activities. Hole 913B is also located nearest to Vesterisbanken (Fig. 1), but the seamount probably was active in the Quaternary (Haase and Devey, 1994).

Site 908 is situated on the Hovgård Ridge, the northern boundary of the Barest Basin. The age of the crust is poorly known. Tectonically the site is located in the area of the large shear zone separating the continental margins of Greenland and Spitsbergen. The recent site and the position at the time of deposition of the one ash layer are similar. The age of the ash layer of Hole $908 \mathrm{~A}$ is relatively uncertain but may have been deposited during the time when the northernmost Tertiary to Quaternary volcanic centers on Spitsbergen and Barents Shelf were active (Fig. 1).

\section{Source Areas}

Four major groups of glass shards can be distinguished based on their chemical composition and stratigraphic position:

1. low-K rhyolites occurring in the late Miocene and on top of the middle Miocene sequence (21 layers in Cores 151-907A-11H to $16 \mathrm{H}, 18 \mathrm{H}, 21 \mathrm{H}$, and $151-908 \mathrm{~A}-17 \mathrm{X}$ ),

2. late Miocene basaltic to dacitic series (layers 2, 4, 5, 7, 17, and 52 in Cores $51-907 \mathrm{~A}-11 \mathrm{H}$ to $14 \mathrm{H}$ and $151-908 \mathrm{~A}-17 \mathrm{X}$ ),

3. late middle Miocene mainly basaltic series (layers 29*, 30, $33 *, 28$, and 40 (*also contain a basaltic-andesitic population) in Cores 151-907 A-15H, $16 \mathrm{H}$ and $18 \mathrm{H}$ ), and

4. early middle Miocene low-K basaltic series (layers $46,47,48$, 49,50 , and 51 in Core $151-907 \mathrm{~A}-21 \mathrm{H}$ ). 
A
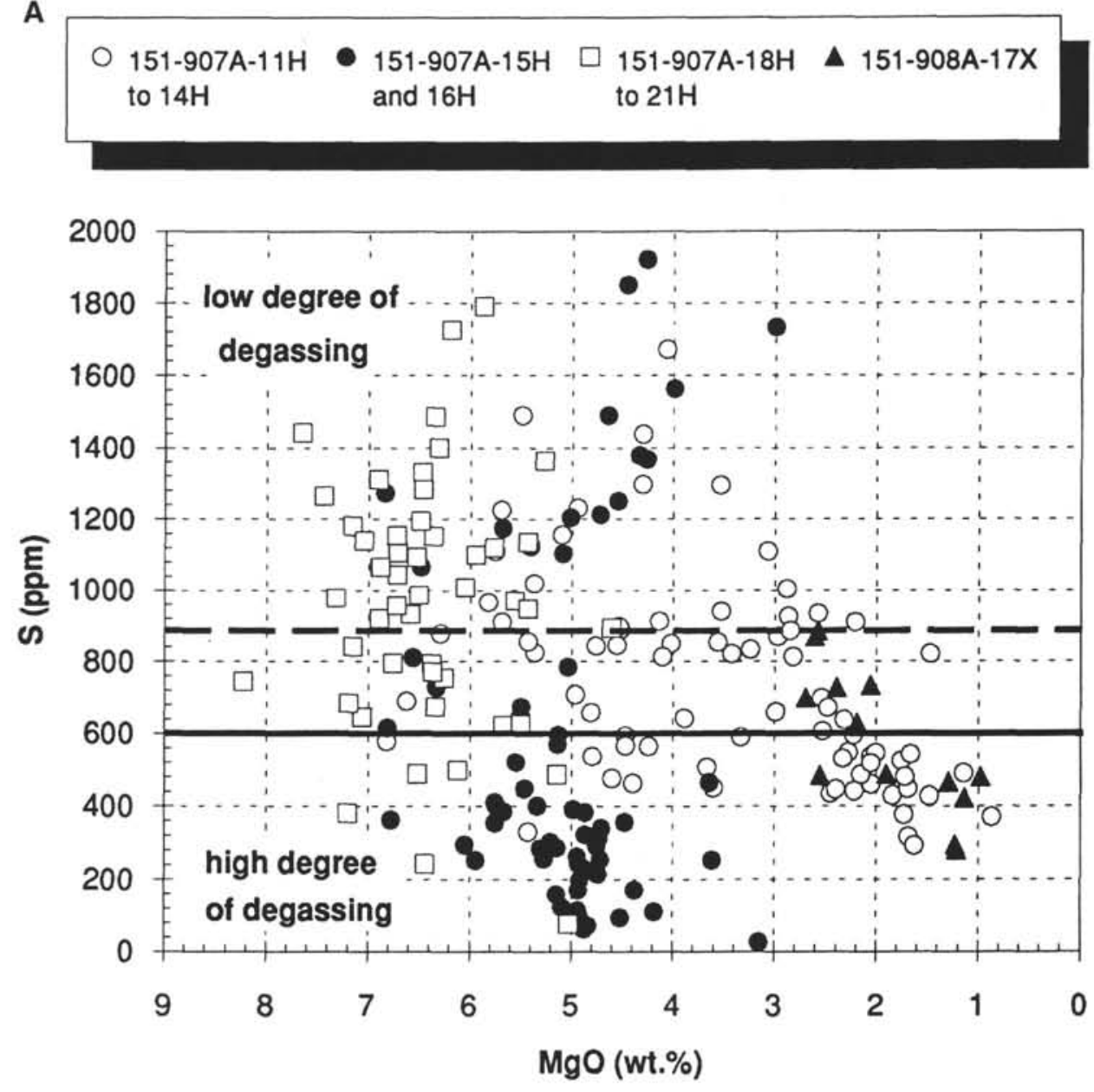

B

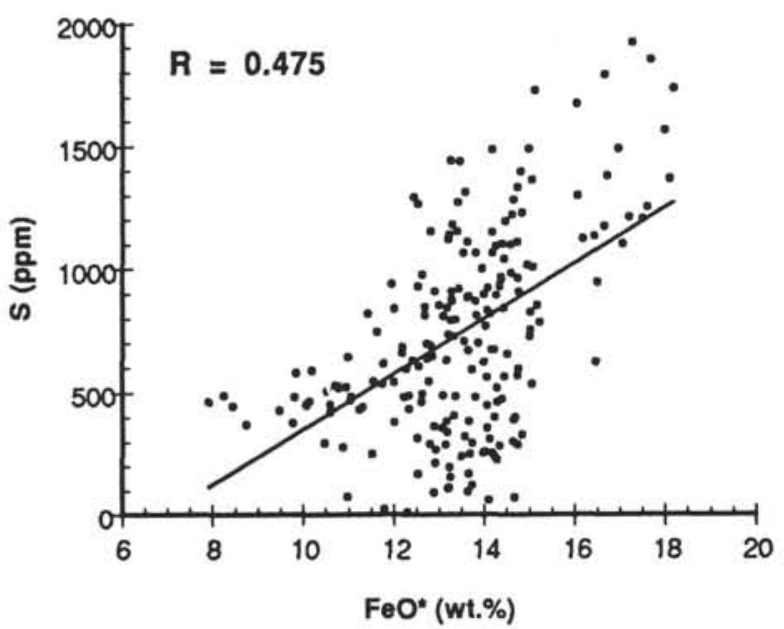

C

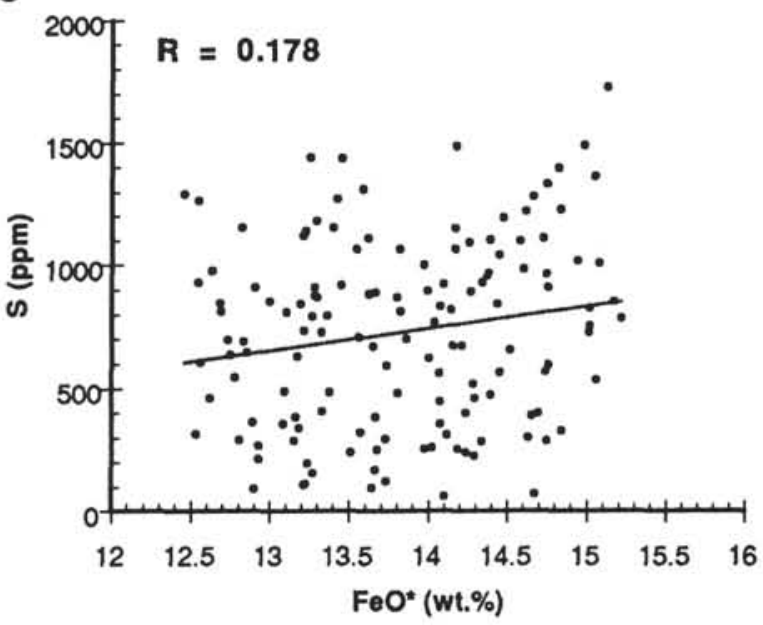

Figure 6. A. Sulfur content of mafic and intermediate glass particles recovered in Hole 907A (Miocene sequence) and 908A. Particles that plot within the field below the unbroken line are significantly degassed with regard to sulfur, whereas sulfur loss was relatively minor from particles that plot above the dashed line. B. Sulfur vs. $\mathrm{FeO} *$ for all analyzed mafic and intermediate glass particles, C. Sulfur vs. $\mathrm{FeO} *$ for mafic and intermediate glass particles with medium $\mathrm{FeO} *$ content (12.5-16 wt\%). 
A

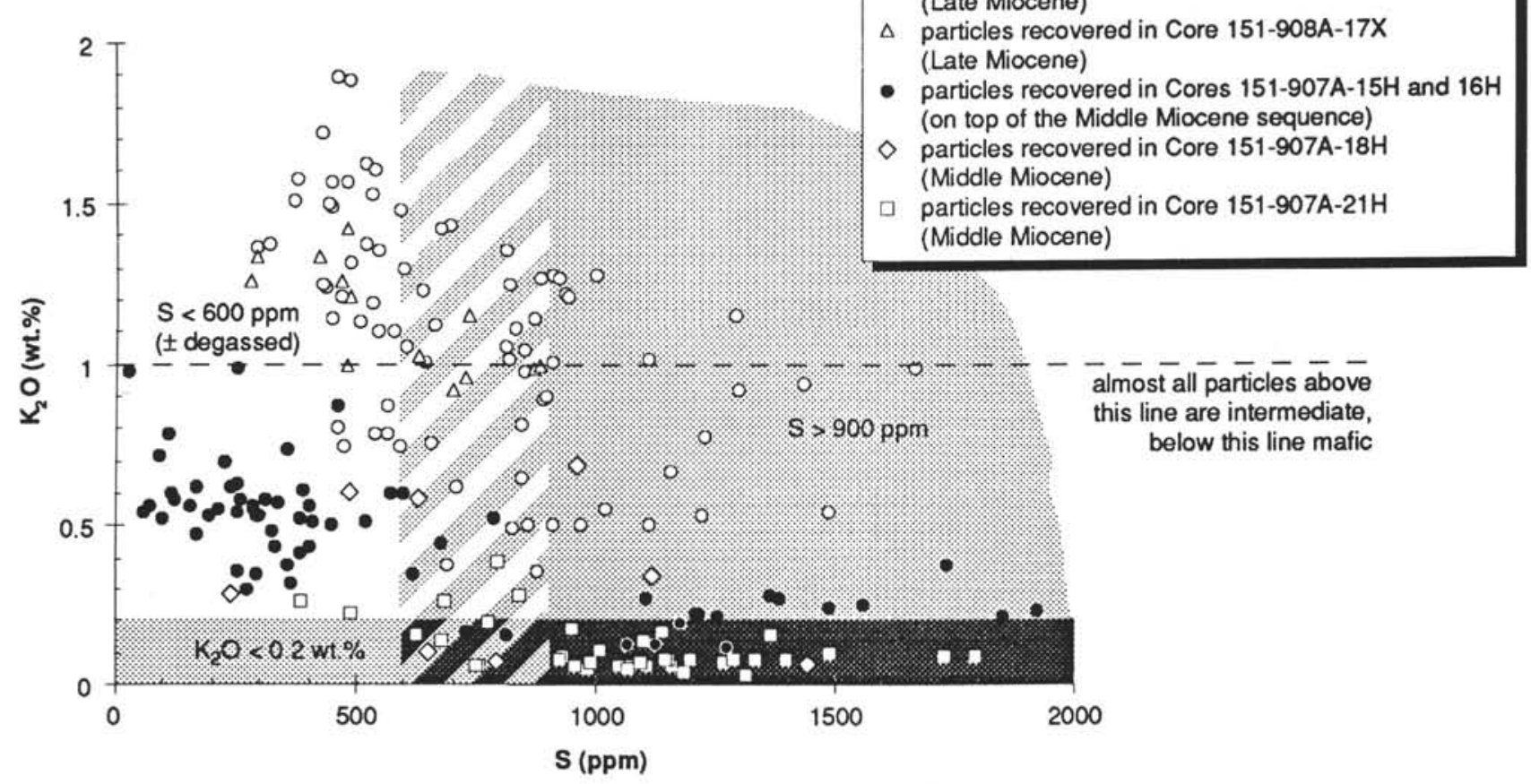

B

field for particles from oldest
ash deposits found in Hole 907A

(layers 46 to 51 in Core 151-907A-21H)

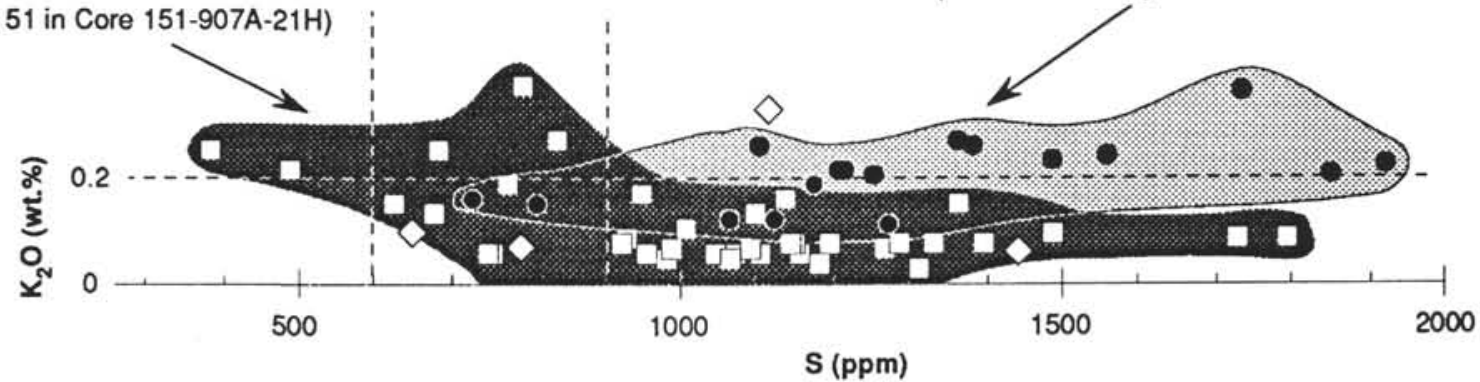

field for particles with $\mathrm{FeO} * 16$ wt.\% (ash-enriched layer 37 in Core 151-907A-16H)

Figure 7. A. Potassium and sulfur content of Miocene basaltic and dacitic glass particles recovered at Leg 151. B. Enlarged detail of (A) showing fields for particles from the oldest ash deposits found in Hole 907A and for particles with high iron content (>16 wt $\% \mathrm{FeO}^{*}$ ).

Additionally, four minor groups occur: middle Miocene high-K alkali rhyolites (layers 45 and 48 in Cores $151-907 \mathrm{~A}-20 \mathrm{H}$ and $21 \mathrm{H}$ ), late Eocene to early Oligocene high-K alkali rhyolites (layer 53 in Core 151-913B-24R), late Miocene trachytes (layer 9 in Core 151$907 \mathrm{~A}-12 \mathrm{H}$ ), and middle Miocene low-K basalts characterized by high iron content (layer 37 in Core 151-907A-16H). No chemical differences are perceptible between the ash deposits recovered at Site 907 and 908.

Iceland is the largest subaerial volcanic area in the North Atlantic and an important source for tephra layers in this region (e.g., Sigurdsson and Loebner, 1981; Bitschene et al., 1989; Bitschene and Schmincke, 1990; Lacasse et al., 1995). The characteristics and, in particular, the chemical composition of the Icelandic volcanic rock series are well known (e.g., Imsland, 1978, 1983; Jakobsson, 1979; Oskarsson et al., 1982; Meyer et al., 1985). All low-K rhyolites have an Icelandic affinity (Fig. 3). Moreover, most analyses match the field for highly evolved rocks of the Tertiary Icelandic central volcanoes Thingmuli and Breiddalur (Fig. 8A; Walker, 1963; Carmichael, 1964). The late Miocene basaltic to dacitic series and the late middle
Miocene basaltic (to andesitic) series also correspond to the mafic and intermediate rocks of these Tertiary Icelandic central volcanoes in their major element compositions (Figs. 8A, B). Furthermore, they clearly follow the Thingmuli tholeiitic trend (Carmichael, 1964) as shown in the $\mathrm{FeO}^{*}$ vs. MgO plot (Fig. 5). Even if the comparison of whole-rock analyses (data from Carmichael and Walker) and analyses of matrix glasses (this study) may be difficult due to the effect of crystallization, the correspondence in concentrations of incompatible elements (e.g., $\mathrm{TiO}_{2}$ and $\mathrm{K}_{2} \mathrm{O}$ ) indicate an Icelandic source area. The average medium diameter of glass particles (about $100 \mu \mathrm{m}$ ) from groups 1 to 3 and prevailing wind directions $(\approx S W$ to NE; Donn and Ninkovich, 1980) also suggest Iceland as a likely source for most of this tephra.

The early middle Miocene low-K basaltic series were probably erupted by submarine eruptions when Site 907 was located close to the axis of the active Kolbeinsey Ridge (see above). The mid-ocean ridges (MOR) in the northern Atlantic produce normal MOR basaltic magmas (Schilling et al., 1983; Neumann and Schilling, 1984; Mertz et al., 1991). Explosive eruptions are uncommon because of the high 


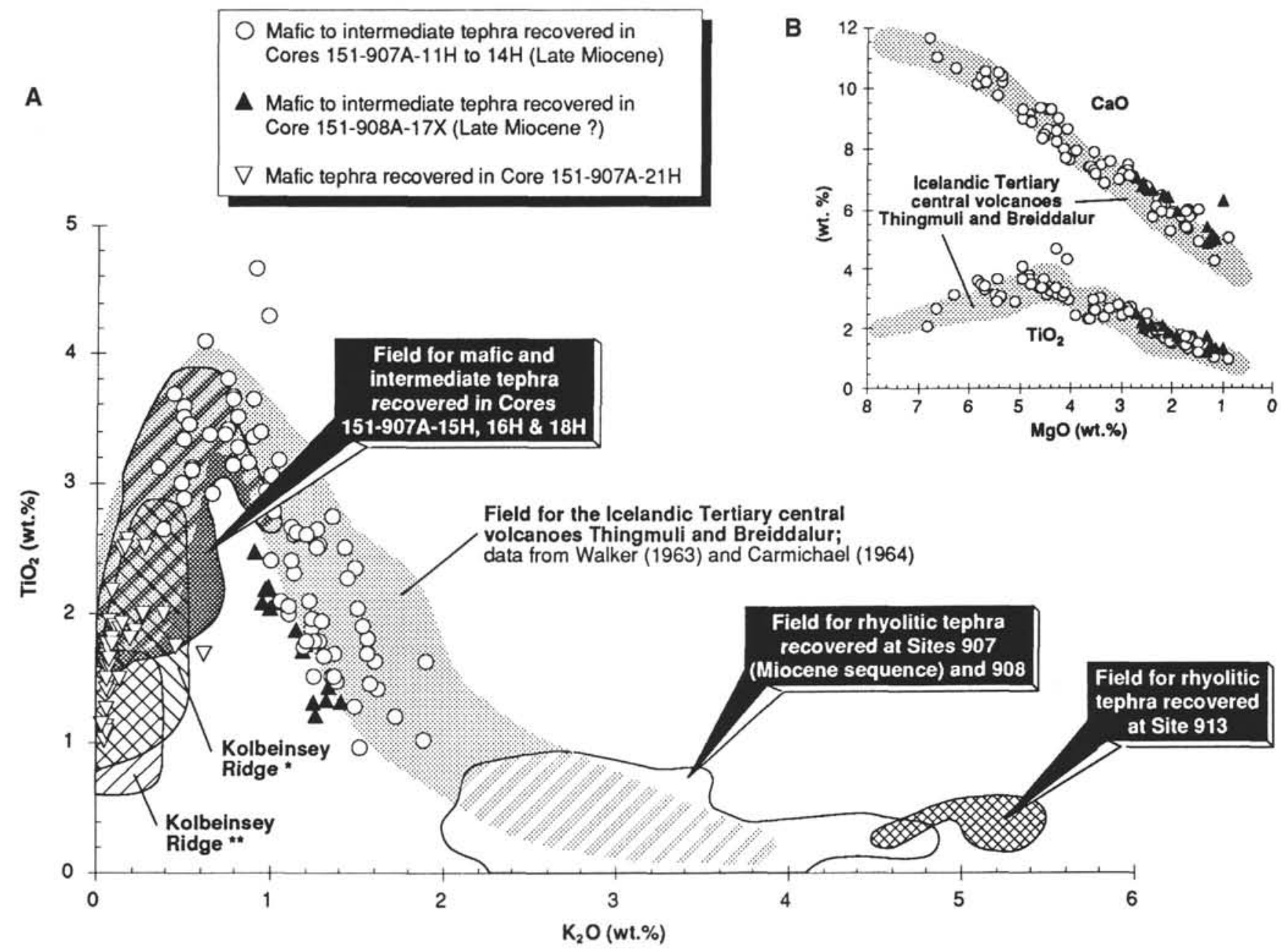

Figure 8. A. $\mathrm{TiO}_{2}$ vs. $\mathrm{K}_{2} \mathrm{O}$ concentrations of rhyolitic and basaltic to dacitic glass particles recovered at Holes $907 \mathrm{~A}$ (Miocene sequence), $908 \mathrm{~A}$, and $913 \mathrm{~B}$. Also shown are fields for two Icelandic Tertiary central volcanoes and Kolbeinsey Ridge basalts (*glass analyses by Lackschewitz and Wallrabe-Adams, 1991 and Lackschewitz et al., 1994; **whole rock analyses by Schilling et al., 1983). B. CaO and $\mathrm{TiO}_{2}$ vs. decreasing $\mathrm{MgO}$ concentrations of late Miocene basaltic to dacitic series recovered at Hole $907 \mathrm{~A}$ and fields for two Icelandic Tertiary central volcanoes.

water depth and low volatile content of MOR basalts. The Kolbeinsey Ridge, as a possible source area of this tephra, is supported by the major element compositions of these series which match the fields of whole-rock analyses by Schilling et al. (1983) or glass analyses by Lackschewitz and Wallrabe-Adams (1991) and Lackschewitz et al. (1994) of Kolbeinsey Ridge tholeiites (Fig. 8A). In particular, the low potassium content of the early middle Miocene basaltic series $(<0.21$ wt\%) are typical for the ridges north and south of Iceland, which are characterized by a decrease in potassium concentrations with increasing distance from Iceland (e.g., Imsland, 1983).

Because of their high alkali content, the late Miocene trachytes are incompatible with an Icelandic source. They show, however, an affinity to Jan Mayen series (Fig. 3), which are generally more alkalic than those of Iceland (Imsland, 1984; Maaløe et al., 1986). Even if Jan Mayen itself seems to have formed within the last $0.5 \mathrm{Ma}$ (Imsland, 1978), eruptive activity is presumed to have occurred in the region of Jan Mayen since late middle Tertiary (e.g., Fitch et al., 1965). This group can therefore be attributed to Jan Mayen; the origin of the other three minor groups listed above remains unclear. Possible source areas for the middle Miocene high- $\mathrm{K}$ alkali rhyolites are Jan Mayen magmatic province (Bitschene et al., 1989) and volcanic centers near the Iceland-Faeroe Ridge (Sigurdsson and Loebner, 1981), where volcanic activity ceased until the middle Miocene. Moreover, these series also show an Icelandic affinity (Fig. 3). Late Eocene to early Oligocene high-K alkali rhyolites (Hole 913B) are too old for an origin at the Jan Mayen magmatic province, but they can be attributed to igneous activity on the East Greenland continental margin and the Iceland-Faeroe Ridge (Sigurdsson and Loebner, 1981) or possibly to the Icelandic province (Proto Iceland?; Eldholm and Thiede, 1980) because of their Icelandic affinity (Fig. 3). Finally, the middle Miocene low-K basalts with high iron content show some typical features for Kolbeinsey Ridge basalts, such as high sulfur concentrations or low potassium content (Fig. 7B), but the maximum $\mathrm{FeO}$ * contents reported from Kolbeinsey Ridge basalts $(<16 \mathrm{wt} \%)$ are $1-2 \mathrm{wt} \%$ less than the composition of this tephra (average $17.2 \mathrm{wt} \%$ ). Also, an Icelandic source is not likely because of the high $\mathrm{FeO}^{*}$ concentrations (e.g., Fig. 5) even if they match the composition of basalts from Tertiary Icelandic central volcanoes for most other major elements.

\section{Volcanic History of the Late and Middle Miocene}

With paleomagnetic ages and sedimentation rates (Shipboard Scientific Party, 1995a) in mind, the excellent recovery (102.6\%) along the late and middle Miocene sequence of Hole 907A allows some 
conclusions on the temporal evolution of volcanism during this time in the North Atlantic region, which has been controversially discussed by previous authors. Sigurdsson and Loebner (1981) inferred, on the basis of Deep Sea Drilling Program (DSDP) Leg 38 cores, a middle Miocene episode of abundant explosive activity and a strong decline in the late Miocene. On the other hand, Donn and Ninkovich (1980) used piston and DSDP cores (Leg 38) to infer a rate of 2.5 deposits/m.y. for the middle Miocene and 7.5 deposits $/ \mathrm{m}$.y. for the late Miocene. These authors caution, however, against overinterpreting their data because of the poor core recovery during Leg 38 . In contrast, Bitschene et al. (1989), using ash deposits from ODP Leg 104, report 26 ash deposits (5-6 per m.y.) for the late Miocene and 40 (calculated) ash deposits (6-8 per m.y.) for the middle Miocene, and they postulate continuous volcanic activity during this time.

In the middle Miocene sequence of Hole 907A, 31 ash deposits were found altogether. Volcanic activity seems to have been relatively constant during this time, with around 8.5 ash deposits/m.y. in the older part and a slight increase to around 11 ash deposits/m.y. between 13 and $11.3 \mathrm{Ma}$. In contrast, only 20 ash deposits (3.2 ash deposits/m.y.) were recovered in the late Miocene sequence. This apparently indicates a strong decrease in volcanic activity in the late Miocene, which does not correspond to increasing spreading rates in the North Atlantic (Talwani and Eldholm, 1977; Sigurdsson and Loebner, 1981) and increasing accumulation rates of basalts in Iceland (Sæmundsson et al., 1980) at the end of the middle Miocene and during the late Miocene, respectively.

This apparent contradiction could be partly explained by a significant change with time in the chemical composition as well as inferred eruption conditions and sources of the ash deposits. During the greater part of the middle Miocene sequence, basaltic ashes clearly dominate over rhyolitic ash. Most of the oldest tephra found in this interval were probably formed in submarine conditions, mainly at the Kolbeinsey Ridge, whereas the younger deposits might be produced in shallow water and subaerial conditions in Iceland. On top of the middle Miocene succession (Core 151-907A-15H), intermediate series with Icelandic affinity occur first and the share of rhyolitic deposits increases. In contrast, during the late Miocene, pure basaltic ash deposits are rare and intermediate series occur frequently but rhyolitic deposits clearly dominate. Nearly all late Miocene ash deposits can be attributed to Icelandic sources and were probably produced by plinian and highly explosive shallow water eruptions, respectively. This change in compositions and possible sources could be caused by the changing position of Site 907 relative to the ridge axis. Whereas, in the early middle Miocene ash particles that were formed, for example, by cooling granulation during effusive submarine eruptions at the ridge could be transported easily by mass flows or small turbidity currents to the ancient location of Site 907 close to ridge, in the late Miocene the majority of those particles did not reach this site because of the larger transport distance between the ridge and the position of the site. It follows from this that the early middle Miocene cores drilled at Site 907A contain both explosively formed tephra and those formed by cooling granulation at the submarine ridge, whereas younger cores contain almost only explosively formed and airborne tephra and hardly any tephra formed by cooling granulation.

The deposition rate in the early middle Miocene, considering exclusively probably airborne tephra erupted by explosive (pyroclastic or hydroclastic) eruptions, is 3.6 deposits/m.y., increases strongly to 9-10 deposits/m.y. in the late middle Miocene, and decreases again to $3.2 /$ deposits/m.y. in the late Miocene. This indicates a rather continuous explosive volcanic activity in the North Atlantic during the middle and late Miocene interrupted by a short, but significant increase in explosive activity during the late middle Miocene. This peak in activity could be related to the onset of the increased spreading rates at Kolbeinsey Ridge, Mohns Ridge, and Iceland in the late middle Miocene.

\section{CONCLUSIONS}

1. The rhyolitic and trachytic tephra deposits can be attributed to highly explosive plinian eruptions. Some ash deposits of mafic and intermediate composition also show the influence of hydroclastic fragmentation processes supporting a shallow submarine or subglacial/sublacustrine eruption environment. The oldest basaltic ashes from Hole $907 \mathrm{~A}$ possibly originate from submarine eruptions (water depth $>200 \mathrm{~m}$ ) as indicated by textural characteristics and their low degree of degassing with regard to sulfur.

2. From stratigraphic considerations, major element chemistry, and the inferred eruption environments, rhyolitic and andesitic ash deposits can be attributed to the Iceland volcanic province; the trachytic tephra probably originated from the Jan Mayen system. The ancient Kolbeinsey Ridge might be the source of the submarine basaltic tephra.

3. The high number of ash deposits in the late Miocene is possibly caused by the ancient location of Site 907 close to the active Kolbeinsey Ridge and subsequent deposition of glass particles formed by cooling granulation at the ridge in addition to airborne tephra. The record of middle to late Miocene fallout deposits indicates a short but strong increase in explosive volcanic activity in the late middle Miocene but similar relatively low rates for the early middle Miocene and late Miocene.

\section{ACKNOWLEDGMENTS}

F. Rack provided valuable information on GRAPE density and magnetic susceptibility measurements, J. Freitag assisted us with the microprobe work, and S. Dorn carried out the grain-size analyses. To them, the Leg 151 Shipboard Scientific Party, and the crew of the Bremen Core Repository we owe thanks. The manuscript was improved by reviews of L. Johnson and two anonymous reviewers. This study was supported by the Deutsche Forschungsgemeinschaft (Grant Th 200/19-1).

\section{REFERENCES}

Bitschene, P.R., and Schmincke, H.-U., 1990. Fallout tephra layers: composition and significance. In Heling, D., Rothe, P., Förstner, U., and Stoffers, P. (Eds.), Sediments and Environmental Geochemistry, Heidelberg (Springer), 48-82.

Bitschene, P.R., Schmincke, H.-U., and Viereck, L., 1989. Cenozoic ash layers on the Vøring Plateau (ODP Leg 104). In Eldholm, O., Thiede, J., Taylor, E., et al., Proc. ODP, Sci. Results, 104: College Station, TX (Ocean Drilling Program), 357-366.

Carmichael, I.S.E., 1964. The petrology of Thingmuli, a Tertiary volcano in eastern Iceland. J. Petrol., 5:435-460.

Donn, W., and Ninkovich, D., 1980. Rate of Cenozoic explosive volcanism in the North Atlantic Ocean inferred from deep sea cores. J. Geophys. Res., 85:5455-5460.

Eldholm, O., 1991. Magmatic-tectonic evolution of a volcanic rifted margin. Mar. Geol., 102:43-61.

Eldholm, O., and Thiede, J., 1980. Cenozoic continental separation between Europe and Greenland. Palaeogeogr., Palaeoclimatol., Palaeoecol., 30:243-259.

Fisher, R.V., and Schmincke, H.-U., 1984. Pyroclastic Rocks: New York (Springer-Verlag).

Fitch, F.J., Grasty, R.L., and Miller, J.A., 1965. Potassium-Argon ages of rocks from Jan Mayen and an outline of its volcanic history. Nature, 207:1349-1351.

Fröhlich, G., Lorenz, V., and Zimanowski, B., 1990. Physical mechanism of lava-water interactions. IAVCEI Internat. Volcanol. Congr. (Abstract) 
Gerlach, T., 1986. Exsolution of $\mathrm{H}_{2} \mathrm{O}, \mathrm{CO}_{2}$ and $\mathrm{S}$ during eruptive episodes at Kilauea Volcano, Hawaii. J. Geophys. Res., 91:12177-12185.

Grönvold, K., and Johannesson, H., 1984. Eruption in Grimsvötn 1983: course of events and chemical studies of the tephra. Jokull, 34:1-11.

Haase, K., and Devey, C., 1994. The petrology and geochemistry of Vesteris seamount, Greenland basin - an intraplate alkaline volcano of non-plume origin. J. Petrol., 35:295-328.

Hunt, J.B., and Hill, P.G., 1993. Tephra geochemistry: a discussion of some persistent analytical problems. The Holocene, 3:271-278.

Imsland, P., 1978. The petrology of Iceland: some general remarks. Nordic Volcanol. Inst. Rep., 7808.

- 1983. Iceland and the ocean floor: comparison of chemical characteristics of the magmatic rocks and some volcanic features. Contrib. Mineral. Petrol., 83:31-37.

,- 1984 . Petrology, mineralogy and evolution of the Jan Mayen magma system. Soc. Scientar. Island., 43.

Jakobsson, S., 1979. Outline of the petrology of Iceland. Jokull, 29:57-73.

Lacasse, C., Sigurdsson, H., Jóhannesson, H., Peterne, M., and Carey, C., 1995. The source of ash zone I in the North Atlantic. Bull. Volcanol., 57$1: 18-32$.

Lackschewitz, K., Dehn, J., and Wallrabe-Adams, H.-J., 1994. Volcaniclastic sediments from mid-oceanic Kolbeinsey Ridge, north of Iceland: evidence for submarine volcanic fragmentation process. Geology, 22:975978.

Lackschewitz, K.S., and Wallrabe-Adams, H.-J., 1991. Composition and origin of sediments on the mid-oceanic Kolbeinsey Ridge, north of Iceland. Mar. Geol., 101:71-82.

Le Bas, M.J., Le Maitre, R.W., Streckeisen, A., and Zanettin, B., 1986. A chemical classification of volcanic rocks based on the total alkali-silica diagram. J. Petrol., 27:745-750.

Maaløe, S., Sorensen, I., and Hertogen, J., 1986. The trachybasaltic suite of Jan Mayen. J. Petrol., 27:439-466.

Mertz, D., Devey, C., Todt, W., Stoffers, P., and Hofmann, A., 1991. Sr-Nd$\mathrm{Pb}$ isotope evidence against plume-asthenosphere mixing north of Iceland. Earth Planet. Sci. Lett., 107:243-255.

Metrich, N., and Clocchiatti, C., 1989. Melt inclusion investigation of the volatile behaviour in historic alkali basaltic magmas of Etna. Bull. Volcanol., 51:185-198.

Metrich, N., Sigurdsson, H., Meyer, P.S., and Devine, J.D., 1991. The 1783 Lakagigar eruption in Iceland: geochemistry, $\mathrm{CO}_{2}$ and sulfur degassing. Contrib. Mineral. Petrol., 107:435-447.

Meyer, P., Sigurdsson, H., and Schilling, J.-G., 1985. Petrological and geochemical variations along Iceland's neovolcanic zones. J. Geophys. Res., 90:10043-10072.

Moore, J., and Fabbi, B., 1971. An estimate of the juvenile sulfur content of basalt. Contrib. Mineral. Petrol., 23:118-127.

Moore, J.G., Batchelder, J.N., and Cunningham, C.G., 1977. $\mathrm{CO}_{2}$-filled vesicles in mid-ocean basalt. J. Volcanol. Geotherm. Res., 2:309-327.

Moore, J.G., and Calk, L.C., 1991. Degassing and differentiation in subglacial volcanoes, Iceland. J. Volcanol. Geotherm. Res., 46:157-180.
Moore, J.G., and Schilling, J.-G., 1973. Vesicles, water, and sulfur in Reykjanes Ridge basalts. Contrib. Mineral. Petrol., 41:105-118.

Mørk, M.B.E., and Duncan, R.A., 1993. Late Pliocene basaltic volcanism on the Western Barents Shelf margin: implications from petrology and ${ }^{40} \mathrm{Ar}$ ${ }^{39} \mathrm{Ar}$ dating of volcaniclastic debris from a shallow drill core. Nor. Geol. Tidsskr., 73: 209-225.

Mutter, J.C., Buck, W.R., and Zehnder, C.M., 1988. Convective partial melting. 1. A model for the formation of thick basaltic sequences during the initiation of spreading. J. Geophys. Res., 93:1031-1048.

Neumann, E.-R., and Schilling, J.-G.E., 1984. Petrology of basalts from the Mohns-Knipovich Ridge, the Norwegian-Greenland. Sea. Contrib. Mineral. Petrol., 85:209-223.

Nunns, A.G., 1983. Plate tectonic evolution of the Greenland-Scotland Ridge and surrounding regions. In Bott, H.P., Saxov, S., Talwani, M., and Thiede, J. (Ed.), Structure and Development of the Greenland-Scotland Ridge: New York (Plenum), NATO Conf. Ser. 4, Mar. Sci., 8:11-30.

Oskarsson, N., Sigvaldason, G.E., and Steinthorsson, S., 1982. A dynamic model of rift zone petrogenesis and the regional petrology of Iceland. $J$. Petrol., 23:28-74.

Sæmundsson, K., Kristjansson, L., McDougall, I., and Watkins, N., 1980. KAr dating, geological and paleomagnetic study of a $5-\mathrm{km}$ lava succession in Northern Iceland. J. Geophys. Res., 85:3628-3646.

Schilling, J.-G., Zajac, M., Evans, R., Johnston, T., White, W., Devine, J.D., and Kingsley, R., 1983. Petrologic and geochemical variations along the Mid-Atlantic ridge from $29^{\circ} \mathrm{N}$ to $73^{\circ} \mathrm{N}$. Am. J. Sci., 283:510-586.

Shipboard Scientific Party, 1995a. Site 907. In Myhre, A.M., Thiede, J., Firth, J.V., et al., Proc. ODP, Init. Repts., 151: College Station, TX (Ocean Drilling Program), 57-111.

, 1995b. Site 908. In Myhre, A.M., Thiede, J., Firth, J.V., et al., Proc. ODP, Init. Repts., 151: College Station, TX (Ocean Drilling Program), 113-158.

, 1995c. Site 913. In Myhre, A.M., Thiede, J., Firth, J.V., et al., Proc. ODP, Init. Repts., 151: College Station, TX (Ocean Drilling Program), 345-382.

Sigurdsson, H., and Loebner, B., 1981. Deep-sea record of Cenozoic explosive volcanism in the North Atlantic. In Self, S., and Sparks, R.S.J. (Eds.), Tephra Studies: Dordrecht (Reidel), 289-316.

Talwani, M., and Eldholm, O., 1977. Evolution of the Norwegian-Greenland Sea. Geol. Soc. Am. Bull., 88:969-999.

Walker, G.P.L., 1963. The Breiddalur central volcano, eastern Iceland. Q. J. Geol. Soc. London, 119:29-63.

Werner, R., 1994. Struktur und Entstehung subglazialer/sublakustrischer Vulkane am Beispiel des Vulkankomplexes Herdubreid/Herdubreidartögl in Island [dissertation]. Christian-Albrechts-Univ. Kiel.

Date of initial receipt: 5 July 1995

Date of acceptance: 20 December 1995

Ms 151SR-123 